\title{
Searching for Galactic hidden gas through interstellar scintillation: results from a test with the NTT-SOFI detector ${ }^{\star}$
}

\author{
F. Habibi ${ }^{1,2}$, M. Moniez ${ }^{1}$, R. Ansari ${ }^{1}$, and S. Rahvar ${ }^{2}$ \\ ${ }^{1}$ Laboratoire de l'Accélérateur Linéaire, IN2P3-CNRS, Université de Paris-Sud, BP 34, 91898 Orsay Cedex, France \\ e-mail: moniez@lal.in2p3.fr \\ 2 Department of Physics, Sharif University of Technology, PO Box, 11365-9161 Tehran, Iran
}

Received 22 June 2010 / Accepted 17 October 2010

ABSTRACT

\begin{abstract}
Aims. Stars twinkle because their light propagates through the atmosphere. The same phenomenon is expected at a longer time scale when the light of remote stars crosses an interstellar molecular cloud, but it has never been observed at optical wavelength. In a favorable case, the light of a background star can be subject to stochastic fluctuations on the order of a few percent at a characteristic time scale of a few minutes. Our ultimate aim is to discover or exclude these scintillation effects to estimate the contribution of molecular hydrogen to the Galactic baryonic hidden mass. This feasibility study is a pathfinder toward an observational strategy to search for scintillation, probing the sensitivity of future surveys and estimating the background level.

Methods. We searched for scintillation induced by molecular gas in visible dark nebulae as well as by hypothetical halo clumpuscules of cool molecular hydrogen $\left(\mathrm{H}_{2}-\mathrm{He}\right)$ during two nights. We took long series of $10 \mathrm{~s}$ infrared exposures with the ESO-NTT telescope toward stellar populations located behind visible nebulae and toward the Small Magellanic Cloud (SMC). We therefore searched for stars exhibiting stochastic flux variations similar to what is expected from the scintillation effect. According to our simulations of the scintillation process, this search should allow one to detect (stochastic) transverse gradients of column density in cool Galactic molecular clouds of order of $\sim 3 \times 10^{-5} \mathrm{~g} / \mathrm{cm}^{2} / 10000 \mathrm{~km}$.

Results. We found one light-curve that is compatible with a strong scintillation effect through a turbulent structure characterized by a diffusion radius $R_{\text {diff }}<100 \mathrm{~km}$ in the B68 nebula. Complementary observations are needed to clarify the status of this candidate, and no firm conclusion can be established from this single observation. We can also infer limits on the existence of turbulent dense cores (of number density $n>10^{9} \mathrm{~cm}^{-3}$ ) within the dark nebulae. Because no candidate is found toward the SMC, we are also able to establish upper limits on the contribution of gas clumpuscules to the Galactic halo mass.

Conclusions. The limits set by this test do not seriously constrain the known models, but we show that the short time-scale monitoring for a few $10^{6}$ star $\times$ hour in the visible band with a $>4 \mathrm{~m}$ telescope and a fast readout camera should allow one to quantify the contribution of turbulent molecular gas to the Galactic halo. The LSST (Large Synoptic Survey Telescope) is perfectly suited for this search.
\end{abstract}

Key words. dark matter - Galaxy: disk - Galaxy: halo - Galaxy: structure - local interstellar matter - ISM: molecules

\section{Introduction}

The present study was made to explore the feasibility of the detection of scintillation effects through nebulae and through hypothetical cool molecular hydrogen $\left(\mathrm{H}_{2}-\mathrm{He}\right)$ clouds. Considering the results of baryonic compact massive objects searches through microlensing (Tisserand et al. 2007; Wyrzykowski et al. 2010; Alcock et al. 2000; see also the review of Moniez 2010), these clouds should now be seriously considered as a possible major component of the Galactic hidden matter. It has been suggested that a hierarchical structure of cold $\mathrm{H}_{2}$ could fill the Galactic thick disk (Pfenniger et al. 1994; Pfenniger \& Combes 1994) or halo (De Paolis et al. 1995; De Paolis et al. 1998), providing a solution for the Galactic hidden matter problem. This gas should form transparent "clumpuscules" of 30 AU size, with an average density of $10^{9-10} \mathrm{~cm}^{-3}$, an average column density of $10^{24-25} \mathrm{~cm}^{-2}$, and a surface filling factor of $\sim 1 \%$. The detection of these structures thanks to the scintillation of background stars would have a major impact on the Galactic dark matter question.

The OSER project (optical scintillation by extraterrestrial refractors) is proposed to search for scintillation of extra-galactic

* This work is based on observations made at the European Southern Observatory, La Silla, Chile. sources through these Galactic - disk or halo - transparent $\mathrm{H}_{2}$ clouds. This project should allow one to detect (stochastic) transverse gradients of column density in cool Galactic molecular clouds on the order of $\sim 3 \times 10^{-5} \mathrm{~g} / \mathrm{cm}^{2} / 10000 \mathrm{~km}$. The technique can also be used for the nebulae science. The discovery of scintillation through visible nebulae should indeed open a new window to investigate their structure.

The feasibility study described here concerns the search for scintillation through known dark nebulae such as B68 (also identified as LDN57), cb131 (also identified as B93 and L328), through a nebula within the Circinus complex (hereafter called Circinus nebula), and also includes a test for hidden matter search toward the SMC. As discussed below, we did not think it very likely to discover a signal, and the main purpose of the test was to predict the sensitivity of a future optical survey from the measurement of the signal sensitivity in infrared and from the estimate of the variable star background level.

\section{The scintillation process}

\subsection{Basics}

Refraction through an inhomogeneous transparent cloud (hereafter called screen), which is described by a 2D phase delay 

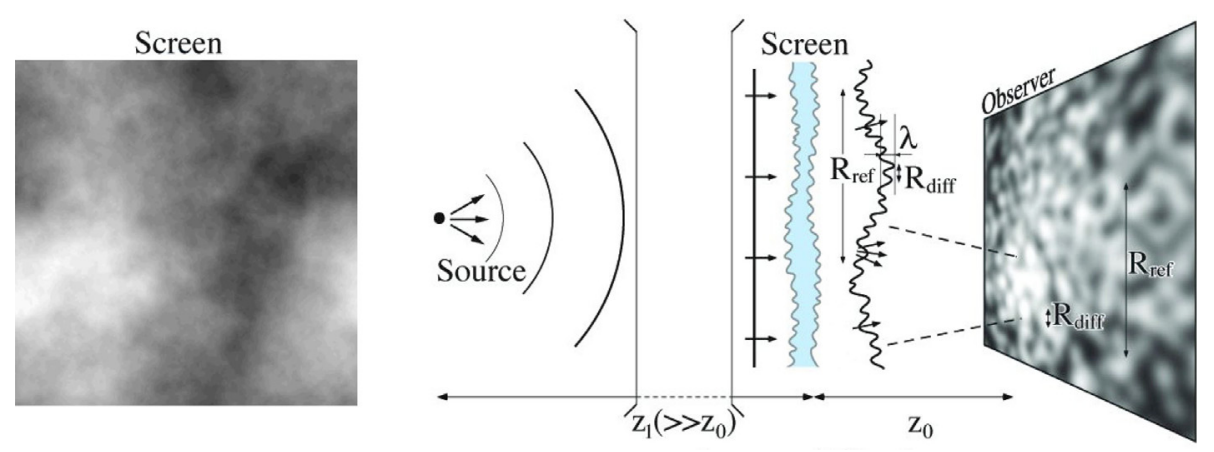

Fig. 1. Left: a 2D stochastic phase screen (gray scale) from a simulation of gas affected by Kolmogorov-type turbulence. Right: the illumination pattern from a point source (left) after crossing such a phase screen. The distorted wavefront produces structures at scales of $\sim R_{\text {diff }}(\lambda)$ and $R_{\text {ref }}(\lambda)$ on the observer's plane.

function $\phi(x, y)$ in the plane transverse to the line of sight, distorts the wave-front of incident electromagnetic waves (Fig. 1; Moniez 2003). The luminous amplitude in the observer's plane after propagation is described by the Huygens-Fresnel diffraction theory. For a point-like, monochromatic source, the intensity in the observer's plane is affected by interferences which take on the speckle aspect in the case of stochastic inhomogeneities. At least two distance scales characterize this speckle, which are related to the wavelength $\lambda$, to the distance of the screen $z_{0}$, and to the statistical characteristics of the stochastic phase delay function $\phi$ :

- The diffusion radius $R_{\text {diff }}(\lambda)$ of the screen, defined as the separation in the screen transverse plane for which the root mean square of the phase delay difference at wavelength $\lambda$ is 1 radian (Narayan 1992). Formally, $R_{\mathrm{diff}}(\lambda)$ is given in a way that $\left\langle\left(\phi\left(x^{\prime}+x, y^{\prime}+y\right)-\phi\left(x^{\prime}, y^{\prime}\right)\right)^{2}\right\rangle=1$ where $\left(x^{\prime}, y^{\prime}\right)$ spans the entire screen plane and $(x, y)$ satisfies $\sqrt{x^{2}+y^{2}}=R_{\text {diff }}(\lambda)$. The diffusion radius characterizes the structuration of the inhomogeneities of the cloud, which are related to the turbulence. As demonstrated in Appendix A, assuming that the cloud turbulence is isotropic and is described by the Kolmogorov theory up to the largest scale (the cloud's width $L_{z}$ ), $R_{\text {diff }}$ can be expressed as

$R_{\mathrm{diff}}(\lambda)=263 \mathrm{~km} \times\left[\frac{\lambda}{1 \mu \mathrm{m}}\right]^{\frac{6}{5}}\left[\frac{L_{z}}{10 \mathrm{AU}}\right]^{-\frac{1}{5}}\left[\frac{\sigma_{3 n}}{10^{9} \mathrm{~cm}^{-3}}\right]^{-\frac{6}{5}}$,

where $\sigma_{3 n}$ is the molecular number density dispersion within the cloud. In this expression, we assume that the average polarizability of the molecules in the medium is $\alpha=0.720 \times$ $10^{-24} \mathrm{~cm}^{3}$, corresponding to a mixing of $76 \%$ of $\mathrm{H}_{2}$ and $24 \%$ of He by mass.

- The refraction radius

$$
R_{\mathrm{ref}}(\lambda)=\frac{\lambda z_{0}}{R_{\mathrm{diff}}} \sim 30860 \mathrm{~km}\left[\frac{\lambda}{1 \mu \mathrm{m}}\right]\left[\frac{z_{0}}{1 \mathrm{kpc}}\right]\left[\frac{R_{\mathrm{diff}}(\lambda)}{1000 \mathrm{~km}}\right]^{-1},
$$

is the size in the observer's plane of the diffraction spot from a patch of $R_{\text {diff }}(\lambda)$ in the screen's plane.

- In addition, long scale structures of the screen can possibly induce local focusing/defocusing configurations that produce long time-scale intensity variations.

\subsection{Expectations from simulation: intensity modulation, time scale}

After crossing an inhomogeneous cloud described by the Kolmogorov turbulence law (Fig. 1, left), the light from a

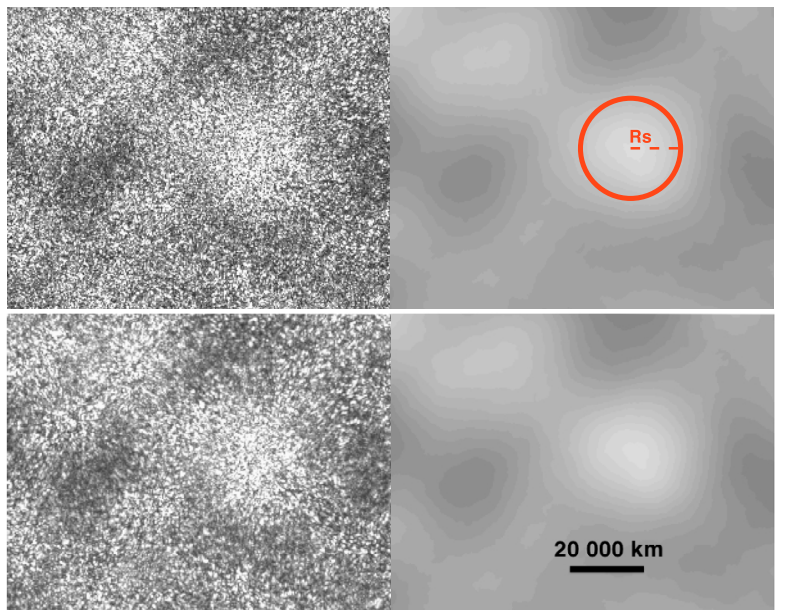

Fig. 2. Simulated illumination map at $\lambda=2.16 \mu \mathrm{m}$ on Earth from a point source (up-left)- and from a K0V star $\left(r_{\mathrm{s}}=0.85 R_{\odot}, M_{V}=5.9\right)$ at $z_{1}=8 \mathrm{kpc}$ (right). The refracting cloud is assumed to be at $z_{0}=160 \mathrm{pc}$ with a turbulence parameter $R_{\text {diff }}(2.16 \mu \mathrm{m})=150 \mathrm{~km}$. The circle shows the projection of the stellar disk (with radius $R_{\mathrm{S}}=r_{\mathrm{s}} \times z_{0} / z_{1}$ ). The bottom maps are illuminations in the $K_{\mathrm{s}}$ wide band $\left(\lambda_{\text {central }}=2.162 \mu \mathrm{m}\right.$, $\Delta \lambda=0.275 \mu \mathrm{m})$.

monochromatic point source produces an illumination pattern on Earth made of speckles of a size $R_{\text {diff }}(\lambda)$ within larger structures of a size $R_{\text {ref }}(\lambda)$ (see Fig. 2, up-left). The illumination pattern from a real stellar source of radius $r_{\mathrm{s}}$ is the convolution of the point-like intensity pattern with the projected intensity profile of the source (projected radius $R_{\mathrm{S}}=r_{\mathrm{S}} \times z_{0} / z_{1}$ ) (Fig. 2, up-right). $R_{\mathrm{S}}$ is then another characteristic spatial scale that affects the illumination pattern from a stellar (not point-like) source.

We simulated these illumination patterns that are caused by the diffusion of stellar source light through various turbulent media as follows:

- we first simulated 2D turbulent screens as stochastic phase delay functions $\Phi(x, y)$, according to the Kolmogorov law ${ }^{1}$; series of screens were generated at $z_{0}=125 \mathrm{pc}$ with $100 \mathrm{~km}<R_{\text {diff }}<350 \mathrm{~km}$;

- we then computed the expected illumination patterns at $\lambda=2.162 \mu \mathrm{m}$ (central wavelength for $K_{\mathrm{s}}$ filter) from diffused background point-like monochromatic sources located at $z_{1}=1 \mathrm{kpc}$ using the Fast Fourier Transform (FFT)

${ }^{1}$ For the Kolmogorov turbulence, the 3D spectral density is a power law relation with exponent $\beta=11 / 3$ (see Appendix A). We also explored power laws with different $\beta$ values and found the same general features. 
technique (see Fig. 2 up-left). Illumination patterns for other wavelengths and geometrical configurations were deduced by simple scaling. In particular, configurations compatible with the structure of the $\mathrm{H}_{2}$ clumpuscules of (Pfenniger et al. $1994)$ were produced for the $J$ passband $\left(R_{\text {diff }}(1.25 \mu \mathrm{m}) \gtrsim\right.$ $17 \mathrm{~km}$, corresponding to clumpuscules with $L_{z}=30 \mathrm{AU}$ and $\sigma_{3 n}<n_{\max }=10^{10} \mathrm{~cm}^{-3}$ )

- we derived series of patterns from diffused extended stellar sources (radii $0.25 R_{\odot}<r_{\mathrm{s}}<1.5 R_{\odot}$ ) by convolution (Fig. 2 top-right);

- finally, we co-added the patterns obtained for the central and the two extreme wavelengths of the $K_{\mathrm{s}}(\Delta \lambda=0.275 \mu \mathrm{m})$ and $J(\Delta \lambda=0.290 \mu \mathrm{m})$ passbands to simulate the diffusion of a wide-band source (Fig. 2 down-right).

The contrast of the patterns are clearly considerably affected by the size of the source (spatial coherence limitation), but only marginally by the bandwidth (temporal coherence).

More details on this simulation will be published in a forthcoming paper (Habibi et al., in prep.).

\subsubsection{Modulation}

In general, the small speckle from a point-source is almost completely smoothed after the convolution by the projected stellar profile, and only the large structures of the size $R_{\text {ref }}(\lambda)$ - or larger - produce a significant modulation. Therefore, as a result of the spatial coherence limitations, the modulation of the illumination pattern critically depends on the angular size of the stellar source $\theta_{\mathrm{s}}=r_{\mathrm{s}} /\left(z_{0}+z_{1}\right) \sim r_{\mathrm{s}} / z_{1}$. Our Monte-Carlo studies show that the intensity modulation index $m_{\text {scint }}=\sigma_{I} / \bar{I}$ decreases when the ratio of the projected stellar disk $R_{\mathrm{S}}$ to the refraction scale $R_{\text {ref }}(\lambda)$ increases, as shown in Fig. 3. This ratio can be expressed as

$\frac{R_{\mathrm{S}}}{R_{\mathrm{ref}}(\lambda)}=\frac{r_{\mathrm{S}} R_{\mathrm{diff}}(\lambda)}{\lambda z_{1}} \sim 2.25\left[\frac{\lambda}{1 \mu \mathrm{m}}\right]^{-1}\left[\frac{r_{\mathrm{s}} / z_{1}}{R_{\odot} / 10 \mathrm{kpc}}\right]\left[\frac{R_{\mathrm{diff}}(\lambda)}{1000 \mathrm{~km}}\right]$.

At the first order, as intuitively expected, the modulation index only depends on this ratio $R_{\mathrm{S}} / R_{\text {ref }}(\lambda)$ and not on other parameters of the phase screen or explicitly on $\lambda$. Indeed, the dispersion of $m_{\text {scint }}$ for series of different configurations generated with the same $R_{\mathrm{S}} / R_{\text {ref }}$ ratio is compatible with the statistical dispersion of $m_{\text {scint }}$ in series of 10 patterns generated with identical configurations. We empirically found that the modulation indices plotted as a function of $R_{\mathrm{S}} / R_{\text {ref }}$ are essentially contained between the curves represented by functions $F_{\min }(x)=0.17 \mathrm{e}^{-1.2 R_{\mathrm{S}} / R_{\text {ref }}}$ and $F_{\text {max }}(x)=0.2 \mathrm{e}^{-0.95 R_{\mathrm{S}} / R_{\text {ref }}}$ in Fig. 3 .

\subsubsection{Time scale}

Because the 2D illumination pattern sweeps the Earth with a constant speed, simulated light-curves of scintillating stars have been obtained by regularly sampling the $2 \mathrm{D}$ illumination patterns along straight lines. For a cloud moving with a transverse velocity $V_{\mathrm{T}}$ with respect to the line of sight, the velocity of the illumination pattern on the Earth is $V_{\mathrm{T}}\left(z_{0}+z_{1}\right) / z_{1} \sim V_{\mathrm{T}}$ as $z_{0} \ll z_{1}$; neglecting the inner cloud evolution - as in radio astronomy (frozen screen approximation (Lyne \& Graham-Smith 1998) the flux variation at a given position is caused by this translation, which induces intensity fluctuations with a characteristic time scale of

$$
\begin{aligned}
t_{\mathrm{ref}}(\lambda) & =\frac{R_{\mathrm{ref}}(\lambda)}{V_{\mathrm{T}}} \\
& \sim 5.2 \min \left[\frac{\lambda}{1 \mu \mathrm{m}}\right]\left[\frac{z_{0}}{1 \mathrm{kpc}}\right]\left[\frac{R_{\mathrm{diff}}(\lambda)}{1000 \mathrm{~km}}\right]^{-1}\left[\frac{V_{\mathrm{T}}}{100 \mathrm{~km} \mathrm{~s}^{-1}}\right]^{-1}
\end{aligned}
$$

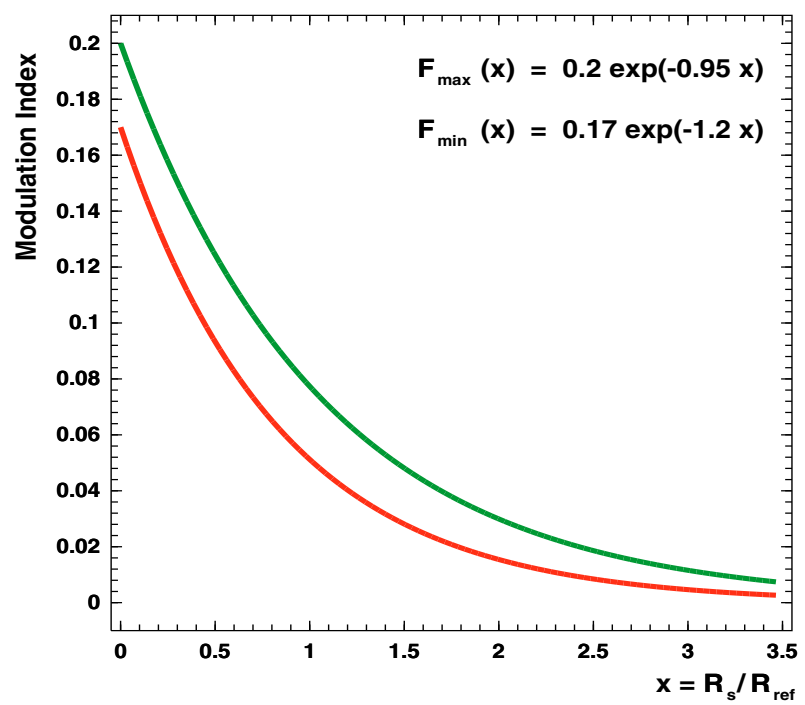

Fig. 3. Expected intensity modulation index $m_{\text {scint }}=\sigma_{I} / \bar{I}$ of illumination patterns from simulated diffused stellar light as a function of $x=R_{\mathrm{S}} / R_{\text {ref }}$. The modulation indices are essentially contained between the curves represented by functions $F_{\min }(x)$ and $F_{\max }(x)$. These functions allow one to constrain $x$ when the constraints on $m_{\text {scint }}$ are known.

Therefore we expect the scintillation signal to be a stochastic fluctuation of the light-curve with a frequency spectrum peaked around $1 / t_{\mathrm{ref}}(\lambda)$ on the order of $(\mathrm{min})^{-1}$.

As an example, the scintillation at $\lambda=0.5 \mu \mathrm{m}$ of a LMC-star through a Galactic $\mathrm{H}_{2}-\mathrm{He}$ cloud located at $10 \mathrm{kpc}$ is characterized by parameter $R_{\mathrm{S}} / R_{\mathrm{ref}}(0.5 \mu \mathrm{m}) \sim\left(r_{\mathrm{S}} / R_{\odot}\right) \times$ $\left(R_{\text {diff }}(0.5 \mu \mathrm{m}) / 222 \mathrm{~km}\right)$. According to Fig. 3 , one expects $m_{\text {scint }}=$ $\sigma_{I} / \bar{I}>1 \%$ if $x=R_{\mathrm{S}} / R_{\text {ref }} \lesssim 2.4\left(=F_{\min }^{-1}(0.01)\right)$. This will be the case for LMC (or SMC) stars smaller than the Sun as soon as $R_{\text {diff }}(0.5 \mu \mathrm{m}) \lesssim 530 \mathrm{~km}$. If the transverse speed of the Galactic cloud is $V_{\mathrm{T}}=200 \mathrm{~km} \mathrm{~s}^{-1}$, the characteristic scintillation time scale will be $t_{\text {ref }} \gtrsim 24 \mathrm{~min}$.

\subsection{Some specificities of the scintillation process}

In the subsections below we briefly describe the properties and specificities of the scintillation signal that should be used to distinguish a population of scintillating stars from the population of ordinary variable objects.

\subsubsection{Chromaticity effect}

Because $R_{\text {ref }}$ depends on $\lambda$, one expects a variation of the characteristic time scale $t_{\text {ref }}(\lambda)$ between the red side of the optical spectrum and the blue side. This property is probably one of the best signatures of the scintillation, because it points to a propagation effect, which is incompatible with any type of intrinsic source variability.

\subsubsection{Relation between the stellar radius and the modulation index}

As shown in Fig. 3, big stars scintillate less than small stars through the same gaseous structure. This characteristic signs the limitations from the spatial coherence of the source and can also be used to statistically distinguish the scintillating population from other variable stars. 


\subsubsection{Location}

Because the line of sight of a scintillating star has to pass through a gaseous structure, we expect the probability for scintillation to be correlated with the foreground - visible gas column-density. Regarding the invisible gas, it may induce clusters of neighboring scintillating stars among a spatially uniform stellar distribution because of foreground - undetected gas structures. This clustering without apparent cause is not expected from other categories of variable stars.

\subsection{Foreground effects, background to the signal}

Conveniently, atmospheric intensity scintillation is negligible through a large telescope $\left(m_{\mathrm{atm}} \ll 1 \%\right.$ for a $>1$ m diameter telescope, Dravins et al. 1997, 1998). Any other atmospheric effect such as absorption variations at the minute scale (because of fast moving cirruses for example) should be easy to recognize as long as nearby stars are monitored together. Asteroseismology, granularity of the stellar surface, spots or eruptions produce variations of very different amplitudes and time scales. A few types of rare recurrent variable stars exhibit emission variations at the minute scale (Sterken \& Jaschek 1996), but they could be identified from their spectrum or type. Scintillation should also not be confused with absorption variations caused by the dust distribution in the cloud; indeed, the relative column density fluctuations needed to produce measurable absorption variations $(\sim 1 \%)$ is higher by several orders of magnitudes than the fluctuations that are able to produce a significant scintillation (only a few $10^{-7}$ for the clumpuscules, and $10^{-3}$ for the Bok globules within a domain of $R_{\text {diff }}$ size).

\subsection{Expected optical depth}

Assuming a Galactic halo completely made of clumpuscules of mass $M_{\mathrm{c}}=10^{-3} M_{\odot}$, their sky coverage (geometrical optical depth) toward the LMC or the SMC should be on the order of $1 \%$ according to Pfenniger et al. (1994). This calculation agrees with a simple estimate based on the density of the standard halo model taken from (Caldwell \& Coulson 1986). Here, we are considering only those structures that can be detected through scintillation. Therefore we quantify the sky coverage of the turbulent sub-structures that can produce this scintillation. We define the scintillation optical depth $\tau_{\lambda}\left(R_{\text {diff max }}\right)$ as the probability for a line of sight to cross a gaseous cloud with a diffusion radius (at $\lambda R_{\text {diff }}(\lambda)<R_{\text {diff max }}$; this optical depth is lower than (or equal to) the total sky coverage of the clumpuscules, because it takes into account only those gaseous structures with a minimum turbulence strength; if $R_{\text {diff }} \rightarrow \infty$, all gaseous structures account for the optical depth and $\tau_{\lambda}(\infty)$ is the total sky coverage of the clumpuscules.

\section{Feasibility studies with the NTT}

As shown in Fig. 3, the search for scintillation induced by transparent Galactic molecular clouds makes it necessary to sample at the sub-minute scale the luminosity of LMC or SMC main sequence stars with a photometric precision of - or better - than $\sim 1 \%$. In principle, this can be achieved with a two meter class telescope with a high quantum efficiency detector and a short dead-time between exposures. To test the concept in a somewhat controlled situation, we also decided to search for scintillation induced by known gas through visible nebulae.
We found that the only setup available for this short timescale search was the ESO NTT-SOFI combination in the infrared, with the additional benefit to enable the monitoring of optically obscured stars that are located behind dark nebulae. The drawback is that observations in infrared do not benefit from the maximum of the stellar emission and the $3.6 \mathrm{~m}$ diameter telescope is barely sufficient to achieve the required photometric precision.

\subsection{The targets}

Considering the likely low optical depth of the scintillation process, all our fields were selected to contain large numbers of target stars. This criterion limited our search to the Galactic plane and to the LMC and SMC fields. For the search through visible nebulae (in the Galactic plane) we added the following requirements:

- maximize the gas column density to benefit from a long phase delay. Data from 2MASS (Skrutskie et al. 2006) where used to select the clouds that induce the stronger reddening of background stars, pointing toward the thickest clouds;

- we selected nebulae that are strongly structured (from visual inspection) and favored those with small spatial scale structures;

- we chose fields that contain a significant fraction of stars that are not behind the nebula to be used as a control sample.

Our targets satisfy these requirements, except for B68, which does not match the second criterion, but was selected so we could benefit from the large number of published studies about this object.

We decided to observe toward the nebulae with the $K_{\mathrm{s}}$ filter to allow the monitoring of highly extincted stars (i.e. behind a high gas column density).

To make a test search for transparent (hidden) gas, we selected a crowded field in the SMC (LMC was not observable at the time of observations). Then we used the $J$ filter to collect the maximum light fluxes attainable with the SOFI detector. Figure 4 shows the four monitored $(4.92 \times 4.92) \operatorname{arcmin}^{2}$ size fields in $R$ and in the $K_{\mathrm{s}}$ or $J$ passbands; Table 1 gives their list and characteristics and also the main observational and analysis information.

Through the cores of B68 and Circinus, gas column densities of $\sim 10^{22}$ atoms $/ \mathrm{cm}^{2}$ induce an average phase delay of $250 \times 2 \pi$ at $K_{\mathrm{s}}$ central wavelength $(\lambda=2.16 \mu \mathrm{m})$. According to our studies, a few percent scintillation signal is expected from stars smaller than the Sun if relative column density fluctuations of only $\sim 10^{-3}$ occur within less than a few thousand kilometers (corresponding to $R_{\text {diff }} \lesssim 2000 \mathrm{~km}$ ). These fluctuations - which are probably rare - could induce dust absorption variations of only $\sim 10^{-3}$, which can be neglected. The expected time scale of the scintillation would be $t_{\text {ref }} \gtrsim 5 \mathrm{~min}$, assuming $V_{\mathrm{T}} \sim 20 \mathrm{~km} \mathrm{~s}^{-1}$.

\subsection{The observations}

During two nights of June 2006 we took a total of 4749 consecutive exposures of $T_{\exp }=10 \mathrm{~s}$, with the infra-red $1024 \times$ 1024 pixel SOFI detector in $K_{\mathrm{s}}(\lambda=2.16 \mu \mathrm{m})$ toward B68, Circinus, cb131, and in $J(\lambda=1.25 \mu \mathrm{m})$ toward the SMC (see Table 1 for details). We recall that for a dedicated search for transparent hidden matter, measuring visible light $(B, V, R$ or $G$ filters) - corresponding to the maximum stellar emission would be better. 

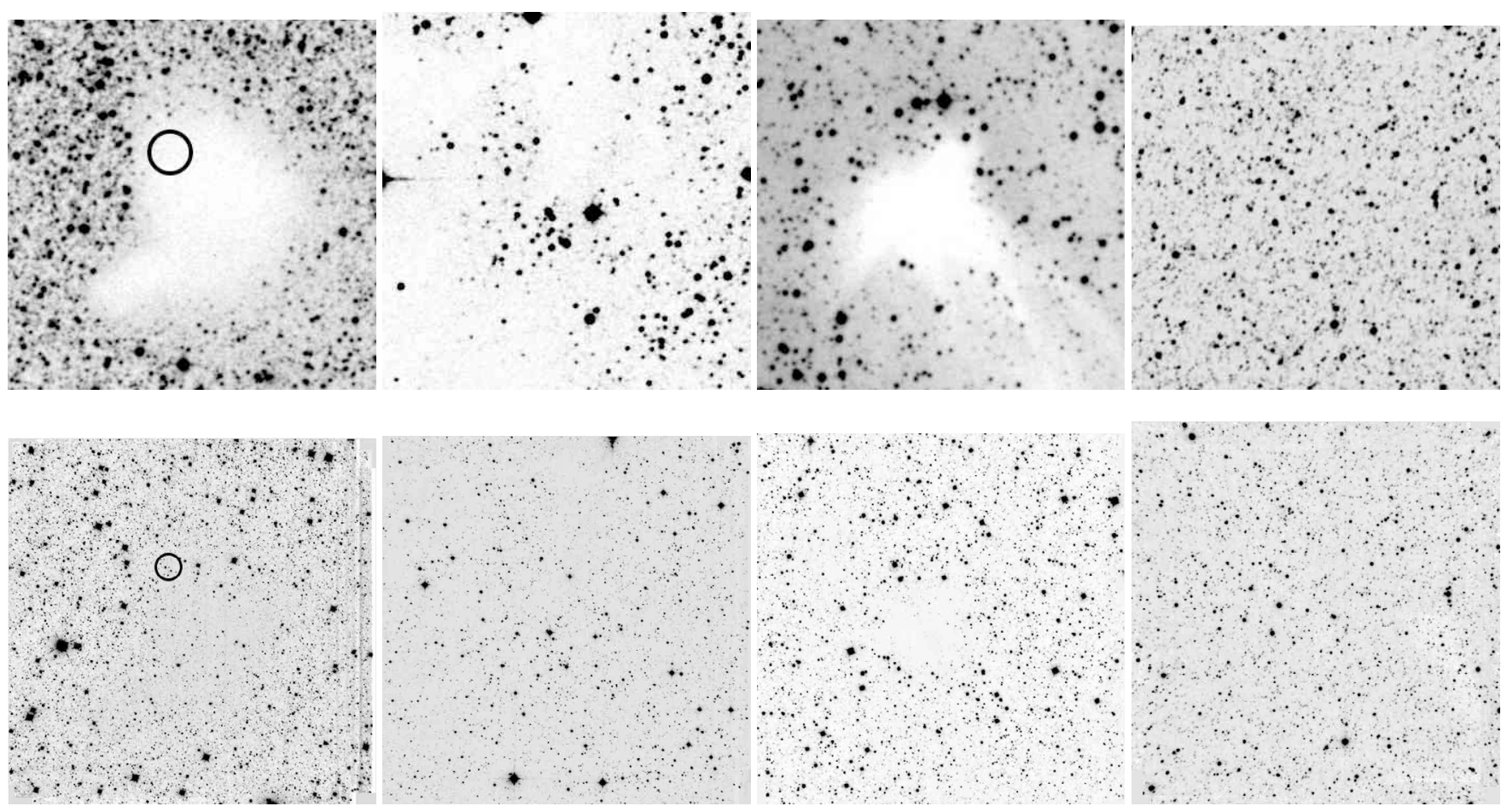

Fig. 4. The four monitored fields, showing the structures of the nebulae and the background stellar densities. From left to right: B68, Circinus, cb131, and the SMC. $U p$ : images from the ESO-DSS2 in R. Down: our corresponding template images (in $K_{\mathrm{s}}$ for B68, cb131 and Circinus, in $J$ for SMC). North is up and East is left. The circles on the B68 images show the position of our selected candidate.

Table 1. Observations and data reduction results.

\begin{tabular}{lcccc}
\hline \hline & SMC & B68 & cb131 & Circinus \\
\hline$\alpha$ (J2000) & $00: 52: 41.3$ & $17: 22: 40.7$ & $18: 16: 59.4$ & $14: 59: 28.9$ \\
$\delta$ (J2000) & $-72: 49: 14.3$ & $-23: 49: 47.2$ & $-18.01: 53.2$ & $-63: 06: 10.1$ \\
central gas density & - & $2.61 \times 10^{5} \mathrm{~cm}^{-3}$ & $1.8 \times 10^{5} \mathrm{~cm}^{-3}$ & \\
center-to-edge density contrast & - & 16.5 & 140. & \\
central column density N1 & - & $2.59 \times 10^{22} \mathrm{~cm}^{-2}$ & $5.8 \times 10^{22} \mathrm{~cm}^{-2}$ & \\
distance of nebula & - & $80 \mathrm{pc}$ & $190 \mathrm{pc}$ & $170 \mathrm{pc}$ \\
minor axis of nebula & - & $17000 \mathrm{AU}$ & $24000 \mathrm{AU}$ & complex \\
distance of sources & $62 \mathrm{kpc}$ & $\sim 8 \mathrm{kpc}$ & $\sim 7 \mathrm{kpc}$ & $\sim 7 \mathrm{kpc}$ \\
\hline light-curve duration night 1 (hours) & 2.18 & 4.77 & 1.25 & 1.84 \\
light-curve duration night 2 & 2.63 & 5.07 & 1.74 & 2.16 \\
\hline number of detected stars & 5042 & 9599 & 9084 & 5249 \\
number of monitored stars & 691 & 1114 & 2779 & 913 \\
magnitude of monitored stars & $J<17.8$ & $K_{\mathrm{s}}<17.1$ & $K_{\mathrm{s}}<17.1$ & $K_{\mathrm{s}}<17.1$ \\
fraction of stars behind dust & $0 \%$ & $46 \%$ & $64 \%$ & - \\
\hline mean number of measurements/star & 980 & 2013 & 629 & 888 \\
\hline
\end{tabular}

Notes. The data on the nebulae are taken from Hotzel et al. (2002) for B68 and from Bacmann et al. (2000) for cb131. The typical distance of the sources in the Galactic plane are taken from Georgelin et al. (1994) and Russeil et al. (1998).

\section{Data reduction}

\subsection{Photometric reduction}

With the EROS software (Ansari 1996) we produced the lightcurves $\phi(t)$ of a few thousands of stars for each target. The reference catalogs of the monitored stars where established from templates obtained through the standard GASGANO procedure (ESO 2001) by co-adding 10 exposures of 60s each for the B68, Circinus, and cb131 fields (13 exposures for the SMC field). The photometric measurements were all aligned with respect to these templates. We experimented with several techniques for the photometric reduction (aperture photometry and Gaussian point spread function (PSF) fitting). We found that the most precise photometric technique, which provides the smallest average point-to-point variation, is the Gaussian PSF fitting. We observed that the Gaussian fit quality of the PSF decreases when the seeing (i.e. the width of the PSF) is small. This effect induces some correlation between the seeing and the estimated flux, and we systematically corrected the flux for this effect, according to a procedure described in Tisserand (2004). Figure 5 shows the dispersion of the measurements along the light-curves. This dispersion depends on the photometric precision and on the - possible but rare - intrinsic stellar apparent variability. We checked that it is not affected by the dust by considering separately a control sample of stars (Fig. 7) that are apart from the nebula (Fig. 5, upper panel). We interpret the lower envelope of Fig. 5 

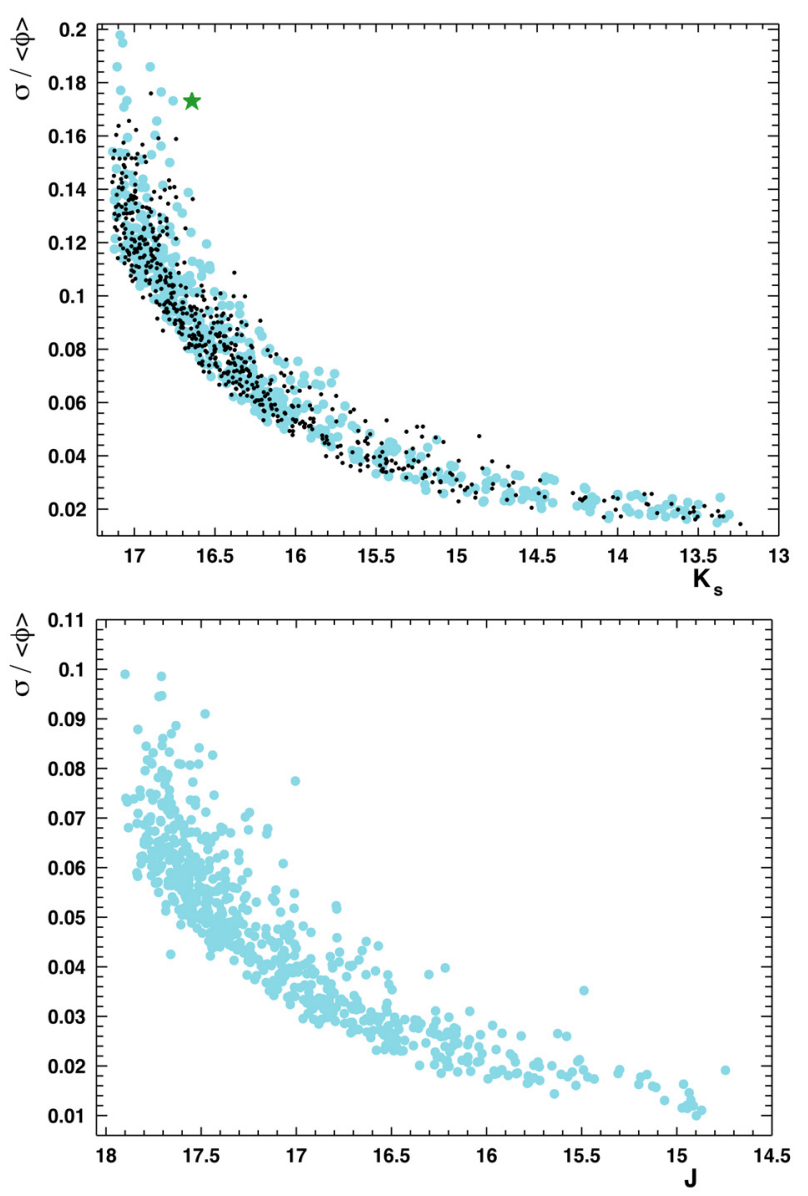

Fig. 5. Dispersion of the photometric measurements along the lightcurves as a function of the mean $K_{\mathrm{s}}$ magnitude (up) and $J$ magnitude (down). Each dot corresponds to one light-curve. In the upper panel, the small black dots correspond to control stars that are not behind the gas; the big blue dots correspond to stars located behind the gas; the big star marker indicates our selected candidate.

as the best photometric precision achieved on (stable) stars at a given magnitude. The outliers that are far above this envelope can be caused by a degradation of the photometric precision in a crowded environment, by the parasitic effect of bad pixels, or by a real variation of the incoming flux. The best photometric precision does not simply result from the Poissonian fluctuations of the numbers of photoelectrons $N_{\gamma \mathrm{e}}$. We discuss the main sources of the precision limitation in Appendix B.

\subsection{Calibration}

The photometric calibration was done using the stars from the 2MASS catalog in our fields (Skrutskie et al. 2006). These stars were found only in the SMC and B68 fields. Because all our fields were observed during the same nights with stable atmospheric conditions, we extrapolated the calibration in the $K_{\mathrm{s}}$ band from B68 to cb131 and Circinus, after checking on series of airmass-distributed images that the airmass differences between the reference images (which are smaller that 0.1 ) could not induce flux variations higher than $0.1 \mathrm{mag}$.

\section{Analysis}

\subsection{Filtering}

We removed the lowest quality images, stars, and mearurements by requiring the following criteria.
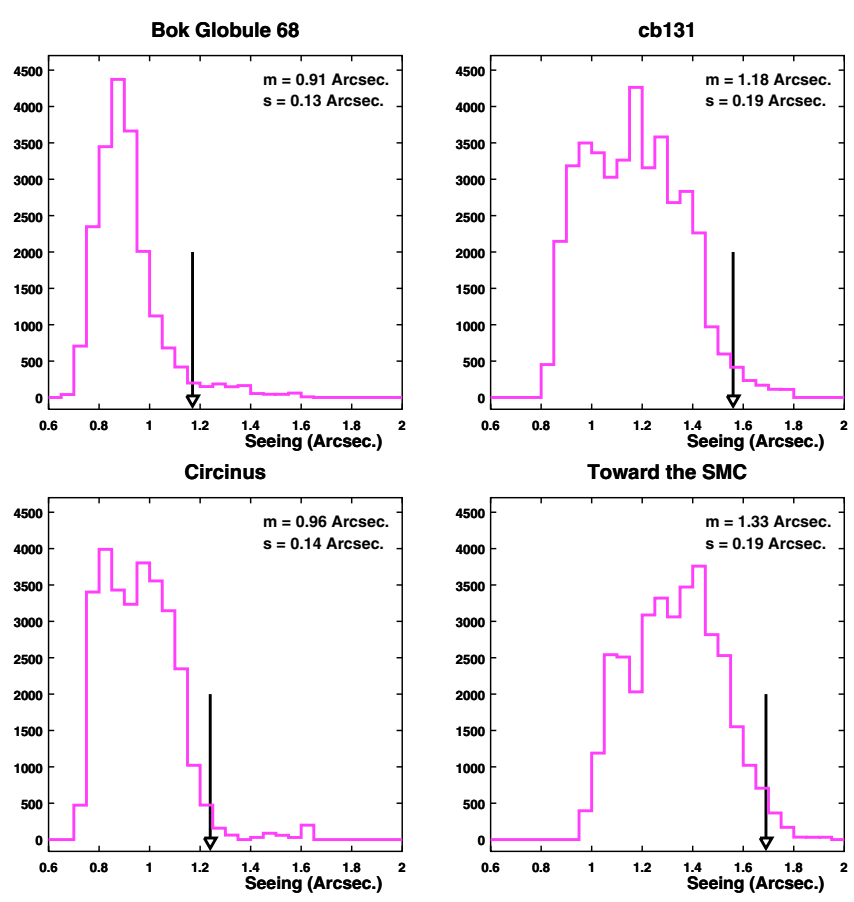

Fig. 6. Seeing distributions of the images towards the four targets. The images with larger seeing than the position of the arrow are discarded.

- Fiducial cuts: we do not measure the luminosity of objects closer than 100 pixels from the limits of the detector to avoid visible defects on its borderlines. The effective size of the monitored fields is then $4.44 \times 4.44 \mathrm{arcmin}^{2}$.

- We remove the images with poor reconstructed seeing by requiring $s<\bar{s}+2 \sigma_{\mathrm{s}}$, where $\bar{s}$ and $\sigma_{\mathrm{s}}$ are the mean and rms of the seeing distributions (see Fig. 6).

- We remove the images with an elongated PSF that have an eccentricity $e>0.55$.

- We reject the measurements with a poor PSF fit quality. This quality varies with the seeing, the filter, the position of the object on the detector, and also with the flux of the star. We require the $\chi^{2}$ of the fit to satisfy $\chi^{2} /$ d.o.f. $<4$.

- Minimum flux: we keep only stars with an instrumental flux $\phi>1000 \mathrm{ADU}$ (corresponding to $J<17.8, K_{\mathrm{s}}<17.1$ ) to allow a reproducibility of the photometric measurements better than $\sim 15 \%$ in $K_{\mathrm{s}}$ and $\sim 8 \%$ in $J$. The number of these stars are given in Table 1 .

- We require at least 10 good quality measurements per night per light-curve before searching for variabilities.

At this stage, we selected a set of sufficiently sampled and well measured light-curves to perform a systematic search for variabilities. The numbers of light-curves we select for each target are given in Table 1. As already mentioned, we divided B68 and cb131 into two subfields containing control stars (out of the nebula's field) and stars behind the nebula (Fig. 7). Table 1 gives the fraction of these stars located behind visible dust, which traces the gas toward B68 and cb131. The case of Circinus is different because we cannot clearly define a control zone.

\subsection{Selecting the most variable light-curves}

We expect the scintillation signal to produce a stochastic fluctuation of the incoming flux with a frequency spectrum peaked around $1 / t_{\mathrm{ref}}(\lambda)$, on the order of $(\mathrm{min})^{-1}$. The point-to-point variations of a stellar light-curve are caused by the photometric 

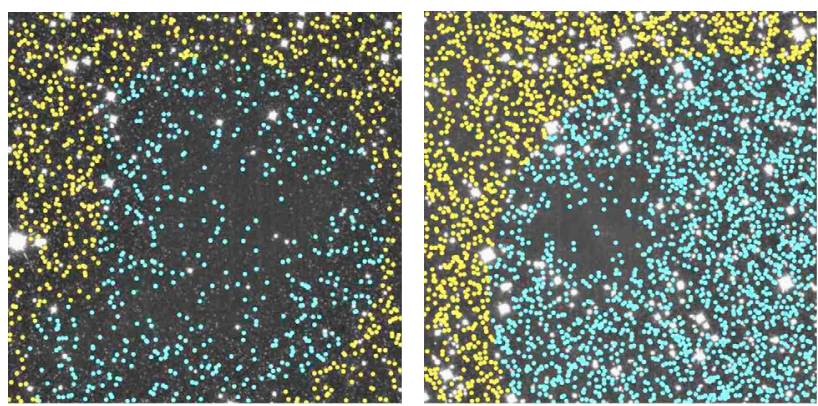

Fig. 7. Definition of the control regions (yellow dots) and the search regions (blue dots) toward B68 (left) and cb131 (right).

uncertainties (statistical and systematical) and by the possible intrinsic incoming flux fluctuations. Note that fluctuations with time scales shorter than the sampling $\left(\delta t \lesssim t_{i+1}-t_{i} \sim 15 \mathrm{~s}\right.$ ) are smoothed in our data, and their high-frequency component cannot be detected. To select a sub-sample that includes the intrinsically most variable objects, we used a simple criterion not specific to a variability type -, which allowed us to both check our ability to detect known variable objects and explore new time domains of variability. Our criterion is based on the ratio $R=\sigma_{\phi} / \sigma_{\text {int }}$ of the light-curve dispersion $\sigma_{\phi}$ to the "internal" dispersion $\sigma_{\text {int }}$, defined as the rms of the differences between the flux measurements and the interpolated values from the previous and next measurements:

$\sigma_{\text {int }}=$

$$
\sqrt{\frac{1}{N_{\text {meas }}} \sum_{i}\left[\phi\left(t_{i}\right)-\left(\phi\left(t_{i-1}\right)+\left[\phi\left(t_{i+1}\right)-\phi\left(t_{i-1}\right)\right] \frac{t_{i}-t_{i-1}}{t_{i+1}-t_{i-1}}\right)\right]^{2}},
$$

where $N_{\text {meas }}$ is the number of flux measurements and $\phi\left(t_{i}\right)$ is the measured flux at time $t_{i}$ (the typical $t_{i}-t_{i-1}$ interval is $\sim 15 \mathrm{~s}$ ). $\sigma_{\text {int }}$ quantifies the point-to-point fluctuations, whereas $\sigma_{\phi}$ is the global dispersion of a light-curve. $R$ is high as soon as there is a correlation between consecutive fluctuations, either because of incoming flux variations or systematic effects. Figure 8 shows the distributions of $R$ versus the apparent magnitude $K_{\mathrm{s}}$ ( $J$ for $\mathrm{SMC})$.

By selecting light-curves with $R>1.6$ (in $K_{\mathrm{s}}$ ) or $R>1.4$ (in $J$ ) - the red dots in Fig. 8 - we retain those with a global variation over the two nights that is significantly larger than the point-topoint variations. Because we found that $R$ almost never exceeds the selection threshold in large series of simulated uniform lightcurves affected by Gaussian errors, we systematically inspected each of the high $R$ objects. We found that almost all of them are artifacts (all the red dots in Fig. 8); we identified the following causes, which are related to the observational conditions:

- during the meridian transit of B68, the light-curves of a series of bright stars located in two regions showed an abrupt flux transition, correlated with the equilibrium change in the mechanics of the telescope;

- some stars looked temporarily brighter because of contamination from the rotating egrets of bright stars;

- we also identified a few stars transiting near hot or dead pixels, which induced a distorsion of the flux determination.

After elimination of these artifacts, only the light-curves marked with a star marker in Fig. 8 remain as reliable variable objects. For this statistically limited test, this simple way of selecting variable objects was sufficient, because we were able to visually inspect each light-curve. We also calculated the autocorrelation
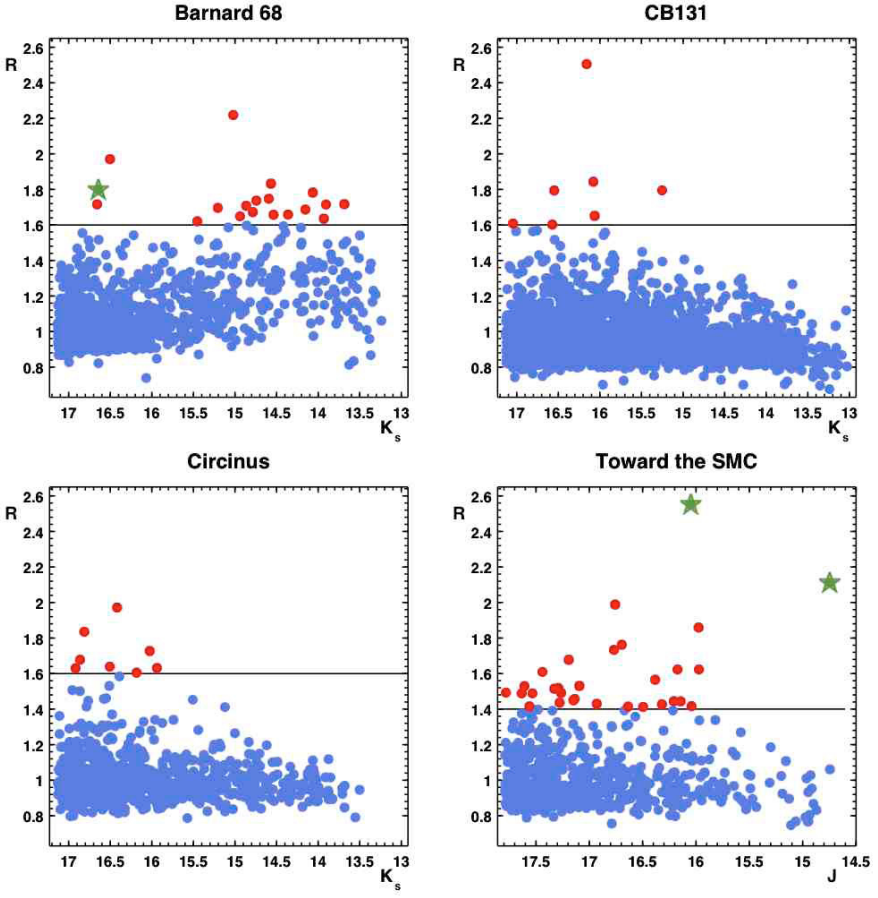

Fig. 8. The $R=\sigma_{\phi} / \sigma_{\text {int }}$ versus $K_{\mathrm{s}}$ (or $J$ ) distributions of the light-curves of the monitored stars. The red dots correspond to the most variable light-curves. The green stars indicate two known variable objects toward SMC and our selected candidate toward B68.

function of the light-curves with no benefit with respect to this basic criterion because of the artifact pollution. For future high statitics observations, we plan to analyze the time power spectra of the light-curves, which should allow an automatic segregation of the artifacts.

\subsection{Sensitivity of the analysis to the known variable objects}

Our selection using variable $R=\sigma_{\phi} / \sigma_{\text {int }}$ is supposed to retain any type of variability as soon as variations occur on a time scale longer than our sampling time. This allowed us to control our sensitivity to known variable stars. This control has been possible only toward the SMC because it is the only field where we found cataloged variable objects. Indeed the CDS and EROS cataloges (Tisserand et al. 2007) contain three variable objects within our SMC-field. We were able to identify two cepheids, $H V 1562\left(\alpha=13.1550^{\circ}, \delta=-72.8272^{\circ}\right.$, J2000, $J=14.7$, periodicity 4.3882 days) and the EROS cepheid $\left(\alpha=13.2250^{\circ}, \delta=-72.7951^{\circ}, \mathrm{J} 2000, J=16.03\right.$, periodicity 2.13581 days). Figure 9 shows the corresponding folded light-curves from the EROS SMC database (phase diagram) (Tisserand et al. 2007; Hamadache et al. 2006; Rahal et al. 2009), on which we superimpose our own observations.

We are content to note that the light-curves of both objects were successfully selected by our analysis; they correspond to the objects marked by a star in Fig. 8 (SMC). Our precision was sufficient to clearly observe the rapidly ascending phase of HV1562 during night 2, as can be seen in Fig. 9.

The third variable object in the SMC field is OGLE SMCSC6 $148139\left(\alpha=13.1446^{\circ}, \delta=-72.8333^{\circ}, \mathrm{J} 2000, B=16.5\right)$. It is a detached eclipsing binary with a periodicity of 1.88508 days. Because no eclipse occured during the data taking, this object was - logically - not selected as a variable by our analysis. 

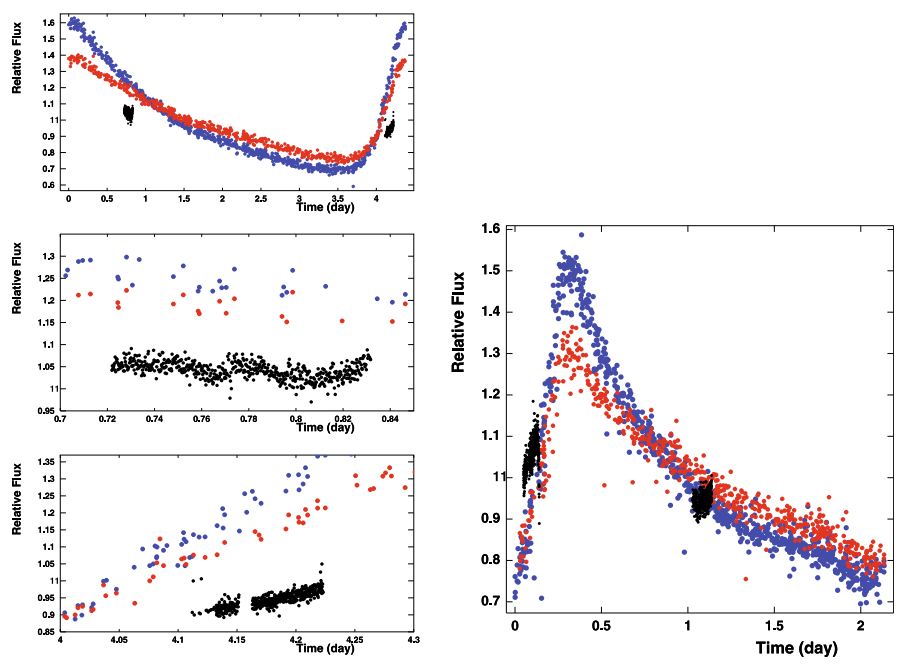

Fig. 9. EROS folded light-curves of cepheids HV1562 (upper-left) and of the EROS object $\left(13.2250^{\circ},-72.7951^{\circ}\right)($ right $)$ in $B_{\text {EROS }}$ and $R_{\text {EROS }}$ passbands, with our NTT observations in $J$ (black dots). The lower-left panels show details around our observations.

\subsection{Signal?}

Only one variable object remains toward B68 after removing all artifacts (see Fig. 10 and see its location $\alpha=260,6762^{\circ}, \delta=$ $-23.8159^{\circ}$ (J2000) in Fig. 4 left). The star has magnitudes $K_{\mathrm{s}}=16.6$ and $J=20.4$; its light is absorbed by dust by $A_{K}=0.99$ magnitude; it is a main sequence star with possible type ranging from $\mathrm{A} 0$ at $9.6 \mathrm{kpc}\left(r_{\mathrm{s}}=2.4 R_{\odot}\right)$ to $\mathrm{A} 5$ at $6.1 \mathrm{kpc}$ $\left(r_{\mathrm{s}}=1.7 R_{\odot}\right)$ or from $\mathrm{F} 0$ at $5.0 \mathrm{kpc}\left(r_{\mathrm{s}}=1.6 R_{\odot}\right)$ to $\mathrm{F} 5$ at $4.0 \mathrm{kpc}$ $\left(r_{\mathrm{s}}=1.4 R_{\odot}\right)$. Consequently, it is a star small enough to experience observable scintillation. The modulation index of the lightcurve is $m=0.17$, which is quite high but not incompatible with a variation owing to scintillation. If we consider that this modulation is caused by a scintillation effect, then it is induced by a turbulent structure with $R_{\mathrm{S}} / R_{\text {ref }}<0.25$, according to Fig. 3; as we know that $r_{\mathrm{s}} / z_{1}>2.5 R_{\odot} / 10 \mathrm{kpc}$, we directly conclude from expression (3) that $R_{\text {diff }}(2.16 \mu \mathrm{m})<96 \mathrm{~km}$; using expression (1), this gives the following constraint on the size and the density fluctuations of the hypothetic turbulent structure:

$$
\sigma_{3 n}>1.45 \times 10^{9} \mathrm{~cm}^{-3}\left[\frac{L_{z}}{17000 \mathrm{AU}}\right]^{-\frac{1}{6}} .
$$

The largest possible outer scale $L_{z}$ corresponds here to the minor axis of B68 (17000 AU), but smaller turbulent structures within the global system may also happen.

A definitive conclusion on this candidate would need complementary multi-epoch and multicolor observations, as explained in Sect. 2.3.1; but the hypothetic turbulent structure that is possibly responsible for scintillation has probably moved from the line of sight since the time of observations, considering its typical size. Nevertheless, reobserving this object would allow one to check for any other type of variability. Considering the short time scale and the large amplitude fluctuations, a flaring or eruptive star may be suspected, but probably not a spotted star or an effect of astroseismology.

An important result comes from the rarity of these fluctuating objects: there is no significant population of variable stars that can mimic scintillation effects, and future searches should not be overwhelmed by background of fakes.

Ideally, for future observation programs aiming for an unambiguous signature, complementary multiband observations

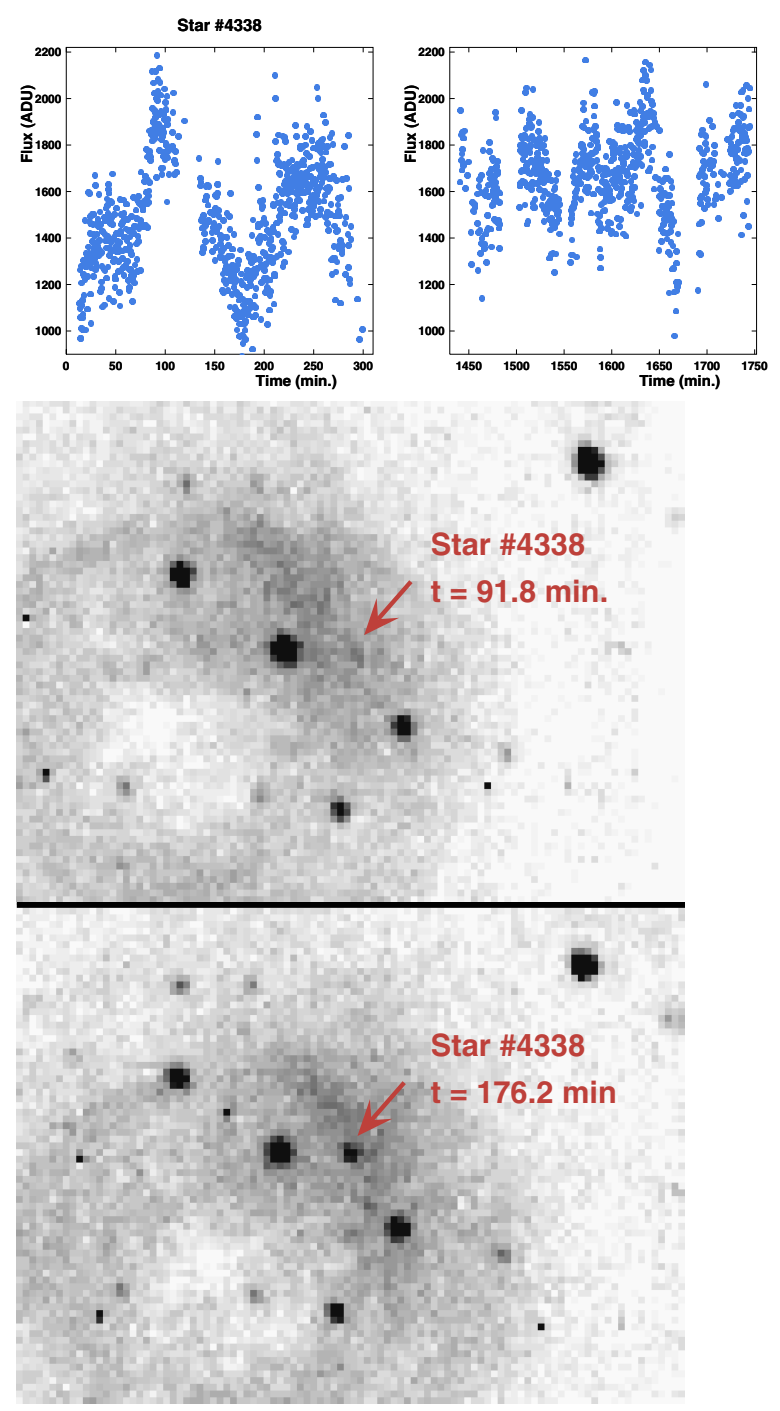

Fig. 10. Light-curves for the two nights of observation (top) and images of the selected candidate toward B68 during a low-luminosity phase (middle) and a high-luminosity phase (bottom); North is up, East is left.

should be planned shortly after the detection of a scintillation candidate. After detection of several candidates, one should also further investigate the correlation between the modulation index and the estimated gas column density to reinforce the scintillation case (see Sects. 2.3.2 and 2.3.3).

\section{Establishing limits on the diffusion radius}

In the following sections we will establish upper limits on the existence of turbulent gas bubbles based on the observed lightcurve modulations. The general technique consists to find the minimum diffusion radius $R_{\text {diff }}$ for each monitored star that is compatible with the observed modulation.

\section{The smallest diffusion radius compatible with observed stellar light-curve fluctuations}

Let us consider a star with radius $r_{\mathrm{s}}$, placed at distance $z_{1}$ behind a screen located at $z_{0}$ (with a projected radius $R_{\mathrm{S}}=r_{\mathrm{s}} \times z_{0} / z_{1}$ ). If a turbulent structure characterized by $R_{\text {diff }}$ (with the corresponding 
$R_{\text {ref }}$ given by Eq. (2)) induces scintillation of the light of this star, the corresponding modulation $m_{\text {scint }}$ is within the limits

$F_{\text {min }}\left(R_{\mathrm{S}} / R_{\text {ref }}\right)<m_{\text {scint }}<F_{\max }\left(R_{\mathrm{S}} / R_{\text {ref }}\right)$,

as predicted from Fig. 3 .

Below we assume that our time sampling is sufficient to take into account any real variation within a time scale longer than a minute. Then, because the observed modulation $m$ of a stellar light-curve results from the photometric uncertainties and from the hypothetic intensity modulation $m_{\text {scint }}$, one can infer that $m_{\text {scint }} \leq m$. This inequality combined with inequality (7) leads to $F_{\min }\left(R_{\mathrm{S}} / R_{\text {ref }}\right)<m$. Because $F_{\min }$ is a decreasing function, it follows that $R_{\mathrm{S}} / R_{\text {ref }}>F_{\min }^{-1}(m)$. Using Eq. (3) and inverting function $F_{\min }(x)=0.17 \mathrm{e}^{-1.2 x}$, this can be expressed as a constraint on the value of $R_{\text {diff }}$ for the gas crossed by the light:

$R_{\mathrm{diff}}>R_{\mathrm{diff}}^{\min } \sim 370 \mathrm{~km}\left[\frac{\lambda}{1 \mu \mathrm{m}}\right]\left[\frac{r_{\mathrm{s}} / z_{1}}{R_{\odot} / 10 \mathrm{kpc}}\right]^{-1} \ln \left[\frac{0.17}{m}\right]$.

\section{Information on the source size}

To achieve a star-by-star estimate of $R_{\text {diff }}^{\min }$, it appears that we need to know $z_{1}$, the distance from the screen to each source, and each source size $r_{\mathrm{s}}$. But because $z_{1} \gg z_{0}$, it follows that $z_{1} \sim z_{0}+z_{1}$, and therefore the knowledge of the angular stellar radius $\theta_{\mathrm{s}}=$ $r_{\mathrm{s}} /\left(z_{0}+z_{1}\right) \sim r_{\mathrm{s}} / z_{1}-$ which can be extracted from the apparent magnitude and the stellar type - is sufficient for this estimate.

We can extract constraints on the stellar apparent radius $\theta_{\mathrm{s}}$ from the $K_{\mathrm{s}}$ (or $J$ ) apparent magnitudes by using the following relation derived from the standard Stefan-Boltzman blackbody law:

$$
\begin{aligned}
& \log \left[\frac{\theta_{\mathrm{s}}}{\theta\left(R_{\odot} \text { at } 10 \mathrm{kpc}\right)}\right]= \\
& 3-\frac{K_{\mathrm{S}}(\text { or } J)}{5}-\frac{\left(V-K_{\mathrm{S}} \text { or } J\right)}{5}+\frac{M_{V \odot}+B C_{\odot}-B C}{5}-2 \log \left[\frac{T}{T_{\odot}}\right],
\end{aligned}
$$

where $R_{\odot} M_{V \odot}, B C_{\odot}, T_{\odot}$ are the solar radius, absolute $V$ magnitude, bolometric correction and temperature; $\theta\left(R_{\odot}\right.$ at $\left.10 \mathrm{kpc}\right)$ is the angular solar radius at $10 \mathrm{kpc} ;\left(V-K_{\mathrm{s}}\right.$ or $\left.J\right)$ taken from Johnson (1966), $B C$ and $T$ taken from Cox (Allen) are the color index, bolometric correction, and temperature respectively that characterize the type of the star (independently of its distance). We use this relation to establish the connection between $K_{\mathrm{s}}$ (or $J$ ) and the angular stellar radius $\theta_{\mathrm{s}}$ for a given stellar type. If the distance to the star is known, then its type is directly obtained from its location in the color-magnitude diagram; if the distance is uncertain, spanning a given domain, we estimate an upper value of $\theta_{\mathrm{s}}$ as follows: for each stellar type, we calculate the distance where the apparent magnitude of the star would be $K_{\mathrm{s}}$. If this distance is within the allowed domain, we estimate $\theta_{\mathrm{s}}$ from Eq. (9). If the branch of the star (main sequence or red giant) is known, we restrain the list of types accordingly. We conservatively consider the maximum value $\theta_{\mathrm{s}}^{\max }$ found with this procedure. In the next two sections, we will use Eq. (8) together with the stellar size constraints for each monitored star to establish distributions of $R_{\text {diff }}^{\min }$ toward the SMC and toward the dark nebulae.

\section{Limits on turbulent Galactic hidden gas toward the SMC}

Here, the distance of the stars are all the same $z_{0}+z_{1} \sim z_{1}=$ $62 \mathrm{kpc}$ (Szewczyk et al. 2009). Therefore the angular stellar radii
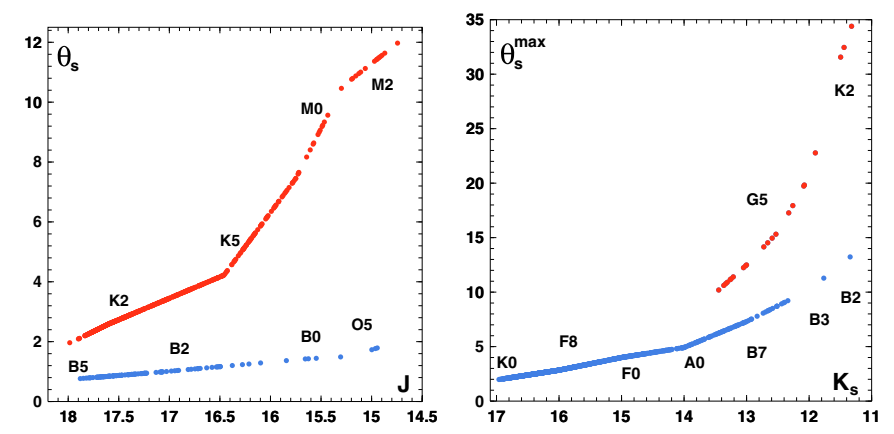

Fig. 11. (Left) Angular radius $\theta_{\mathrm{s}}$ and type of the SMC stars as a function of their apparent magnitude $J$ (in units of the angular radius of the Sun at $10 \mathrm{kpc}$ ). (Right) Maximum angular radius $\theta_{\mathrm{s}}^{\max }$ and corresponding type of the B68 stars as a function of the apparent magnitude $K_{\mathrm{s}}$. The blue lines belong to the main sequence stars, the red lines belong to the red giant stars.

$\theta_{\mathrm{s}}$ can be (roughly) estimated from the observed $J$ magnitude. We used the EROS data (Tisserand et al. 2007; Hamadache et al. 2006; Rahal et al. 2009) to distinguish between main sequence and red giant branch stars from the $R_{\mathrm{EROS}}$ versus ( $B_{\mathrm{EROS}}-R_{\mathrm{EROS}}$ ) color-magnitude diagram; then the stellar type is obtained from the absolute magnitude $M_{J}=J-5 \cdot \log (62000 \mathrm{pc} / 10 \mathrm{pc})$ and $\theta_{\mathrm{s}}$ is derived from Eq. (9) (see Fig. 11, left). With these ingredients, we can estimate from Eq. (8) $R_{\text {diff }}^{\min }$ the lowest value of $R_{\text {diff }}$ compatible with the measured modulation index $m$ for each stellar light-curve. Figure 12a shows the cumulative distribution of this variable $R_{\text {diff }}^{\min }$, that is $N_{*}\left(R_{\mathrm{d}}\right)$, the number of stars whose line of sight do not cross a gaseous structure with $R_{\mathrm{diff}}<R_{\mathrm{d}}$. The bimodal shape of this distribution is caused by the prominence of the red giants in the monitored population: scintillation is expected to be less contrasted for red giant stars because of their large radius; therefore, structures with $R_{\text {diff }} \gtrsim 250 \mathrm{~km}$ cannot induce detectable modulation in the red giant light-curves, and the $R_{\text {diff }}^{\min }$ values are lower than $250 \mathrm{~km}$.

The distribution vanishes beyond $R_{\mathrm{d}} \sim 800 \mathrm{~km}$ because our limited resolution on the main sequence stars (a few \%) prevents us from detecting any scintillation of gaseous structures with $R_{\text {diff }}>800 \mathrm{~km}$. The possible $R_{\text {diff }}$ domain for the hidden gas clumpuscules expected from the model of (Pfenniger et al. 1994; Pfenniger \& Combes 1994) and their maximum contribution to the optical depth are indicated by the gray zone; the minimum $R_{\text {diff }}(\sim 17 \mathrm{~km}$ at $\lambda=1.25 \mu \mathrm{m})$ for these objects is estimated from Eq. (1) assuming the clumpuscule's outer scale is $30 \mathrm{AU}$ and considering the maximum possible value of the density fluctuation, mathematically limited by the maximum density $\left(\sigma_{3 n}^{\max }<n_{\max }=10^{10} \mathrm{~cm}^{-3}\right)$ (Pfenniger et al. 1994; Pfenniger \& Combes 1994).

From the distribution of $N_{*}\left(R_{\mathrm{d}}\right)$, one can infer limits on the scintillation optical depth $\tau_{1.25} \mu \mathrm{m}\left(R_{\mathrm{d}}\right)$ as it is defined in Sect. 2.5. Indeed $N_{*}\left(R_{\mathrm{d}}\right)$ is the number of lines of sights (1.o.s.) that do not cross structures with $R_{\text {diff }}<R_{\mathrm{d}}$. Defining $N_{\text {behind }}$ as the total number of monitored l.o.s. through the nebula (all monitored stars in the present case because we are searching for invisible gas), the upper limit on the optical depth $\tau_{1.25} \mu \mathrm{m}\left(R_{\mathrm{d}}\right)$ is the upper 

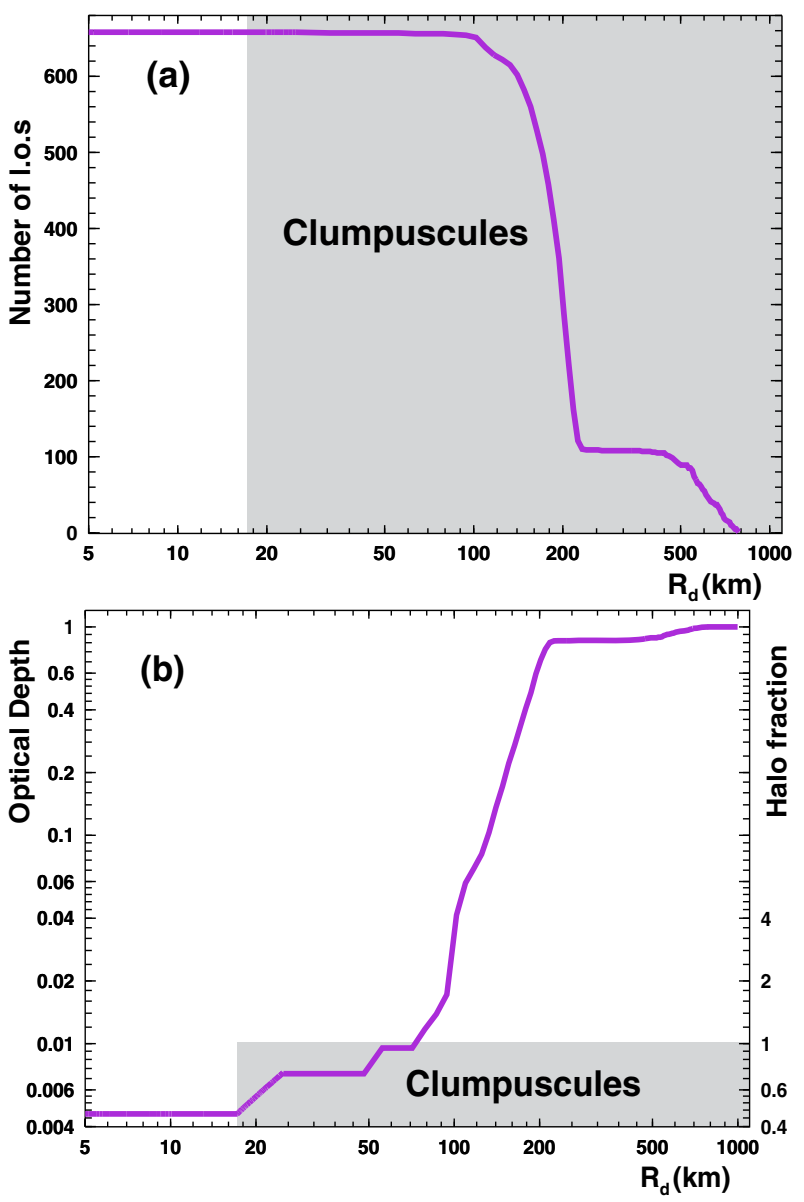

Fig. 12. a) $N_{*}\left(R_{\mathrm{d}}\right)$, the number of SMC stars (or lines of sight) with no turbulent structure of $R_{\mathrm{diff}}(1.25 \mu \mathrm{m})<R_{\mathrm{d}}$ along the line of sight. The gray band shows the allowed $R_{\text {diff }}(1.25 \mu \mathrm{m})$ region for clumpuscules (see text). b) The 95\% CL maximum optical depth of structures with $R_{\mathrm{diff}}<R_{\mathrm{d}}$ toward the SMC. The right scale gives the maximum contribution of structures with $R_{\text {diff }}(1.25 \mu \mathrm{m})<R_{\mathrm{d}}$ to the Galactic halo (in fraction); the gray zone gives the possible region for the hidden gas clumpuscules.

limit on the ratio $p=\frac{N_{\text {behind }}-N_{*}\left(R_{\mathrm{d}}\right)}{N_{\text {behind }}}$. The 95\% statistical upper limit on $p$ is given by the classical confidence interval i.e. ${ }^{2}$ :

- if $N_{*}\left(R_{\mathrm{d}}\right)$ and $N_{\text {behind }}-N_{*}\left(R_{\mathrm{d}}\right)$ are both larger than 4 , then

$$
\tau_{\lambda}\left(R_{\mathrm{d}}\right)<p+1.643 \sqrt{\frac{p(1-p)}{N_{\text {behind }}}} ;
$$

- if $N_{*}\left(R_{\mathrm{d}}\right) \leq 4$, then

$$
\tau_{\lambda}\left(R_{\mathrm{d}}\right)<\frac{N_{\text {behind }}-N_{95 \%}\left(R_{\mathrm{d}}\right)}{N_{\text {behind }}},
$$

where $N_{95 \%}\left(R_{\mathrm{d}}\right)$ is the $95 \% \mathrm{CL}$ Poissonian lower limit on $N_{*}\left(R_{\mathrm{d}}\right)$;

- if $N_{\text {behind }}-N_{*}\left(R_{\mathrm{d}}\right) \leq 4$, then

$$
\tau_{\lambda}\left(R_{\mathrm{d}}\right)<\frac{\left[N_{\text {behind }}-N_{*}\left(R_{\mathrm{d}}\right)\right]_{95 \%}}{N_{\text {behind }}},
$$

where $\left[N_{\text {behind }}-N_{*}\left(R_{\mathrm{d}}\right)\right]_{95 \%}$ is the $95 \%$ CL Poissonian upper limit on $N_{\text {behind }}-N_{*}\left(R_{\mathrm{d}}\right)$.

\footnotetext{
2 The first formula gives the upper limit of the 90\% CL interval for the $p$ value (see classical texbooks like Ventsel 1973); therefore the probability that the true $p$ value is higher than the upper limit of this interval is $5 \%$.
}

As an example, the upper limit on the optical depth for structures with $R_{\text {diff }}<400 \mathrm{~km}$ is obtained from the value of $N_{*}(400 \mathrm{~km}) \sim$ 100 ; as $N_{\text {behind }}=691$, it comes $p \sim 0.86$. For this example, the 95\% CL upper limit on $\tau_{1.25} \mu \mathrm{m}(400 \mathrm{~km})$ is found to be 0.88 from Eq. (10).

The expected optical depth is proportional to the total mass of gas. In Pfenniger et al. (1994) the clumpuscules are expected to cover less than $\sim 1 \%$ of the sky; this means that the maximum optical depth should be $\sim 0.01$ assuming a Galactic halo completely made of gaseous clumpuscules. Therefore, we can interpret our optical depth limit as the upper limit of the contribution of turbulent gaseous structures with $R_{\mathrm{diff}}(1.25 \mu \mathrm{m})<R_{\mathrm{d}}$, expressed in fraction of the halo (right scale in Fig. 12b).

Our upper limit does not yet seriously constrain the model with clumpuscules, but we can extrapolate these results to define a strategy to reach a significant sensitivity. Our monitored stellar population is dominated by red giant stars that would give a lower scintillation signal than smaller stars from the main sequence. This could only be compensated by achieving an excellent photometric precision on the red giants. An easier way to improve a hypothetic signal would be to use $V$ passband instead of $J$. In our test, the use of $J$ filter was imposed by the choice of the SOFI detector, the only one available with a fast readout. According to the ESO exposure time calculator (ESOSOFI 2007), the same precision we obtained in $J$ for the red giants can be reached for $A 0$ stars in $V$ with the same exposure time $(10 \mathrm{~s})$. Therefore, we can extrapolate that an exposure of $\sim 10^{6}$ star $\times$ hour (about 100 times more than our test) obtained with the same type of telescope (NTT) using the $V$ passband (around the maximum of stellar emission) should provide enough sensitivity to significantly constrain the turbulent gas component of the Galactic halo.

\section{Limits on the gas structuration in the nebulae}

For our study of known nebulae, the distance $z_{0}$ to the gas is known, but not the star-by-star $z_{1}$ distances. We first make the hypothesis that the stellar population behind the clouds (whose light is absorbed and diffracted) is the same as the population which is not - or much less - obscured (the so-called control population, see Fig. 7). Toward B68 the red giant stars are distinguished from the main sequence stars with our own $J$ image, also taken with the NTT-SOFI detector, through the $K_{\mathrm{s}}$ versus $\left(J-K_{\mathrm{s}}\right)$ diagram. Using Eq. (9), we established the $K_{\mathrm{s}}$ to $\theta_{\mathrm{s}}^{\max }$ relation, providing the maximum angular radius of a main sequence or red giant star located beyond $4 \mathrm{kpc}$ with $K_{\mathrm{s}}$ apparent magnitude (Fig. 11 right). We conservatively use this angular radius in Eq. (8) to estimate $R_{\text {diff }}^{\mathrm{min}}$. The study of the population behind the dust is complicated by the fact that the stars are obscured. In the case of B68 we were able to correct the apparent magnitudes for the absorption in $K_{\mathrm{s}}$ band; we used the $A_{V}$ absorption map from (Alves et al. 2001) and the relation $A_{K} / A_{V}=0.089$ (Ojha et al. 2000; Glass et al. 1999) to extract $A_{K}$ and deduce the dereddened magnitudes $K_{\mathrm{s}}$. Using the relation of Fig. 11 (right) with these corrected magnitudes, we extract $\theta_{\mathrm{s}}^{\max }$ for each star behind the dust.

Because there is no absorption map for bc131 and the Circinus nebula, we conservatively used the highest possible value of $\theta_{\mathrm{s}}^{\max } \sim 13 \times \theta\left(R_{\odot}\right.$ at $10 \mathrm{kpc}$ ) (corresponding to a B3 type star at $4.5 \mathrm{kpc}$ ).

Figure 13 shows the cumulative $R_{\mathrm{diff}}^{\mathrm{min}}$ distributions and the upper limits on $\tau_{2.16 \mu \mathrm{m}}\left(R_{\mathrm{d}}\right)$ for the obscured regions of B68 and bc131 (the search regions from Fig. 7) and over the complete field of the Circinus nebula, which has indistinct boundaries. The 

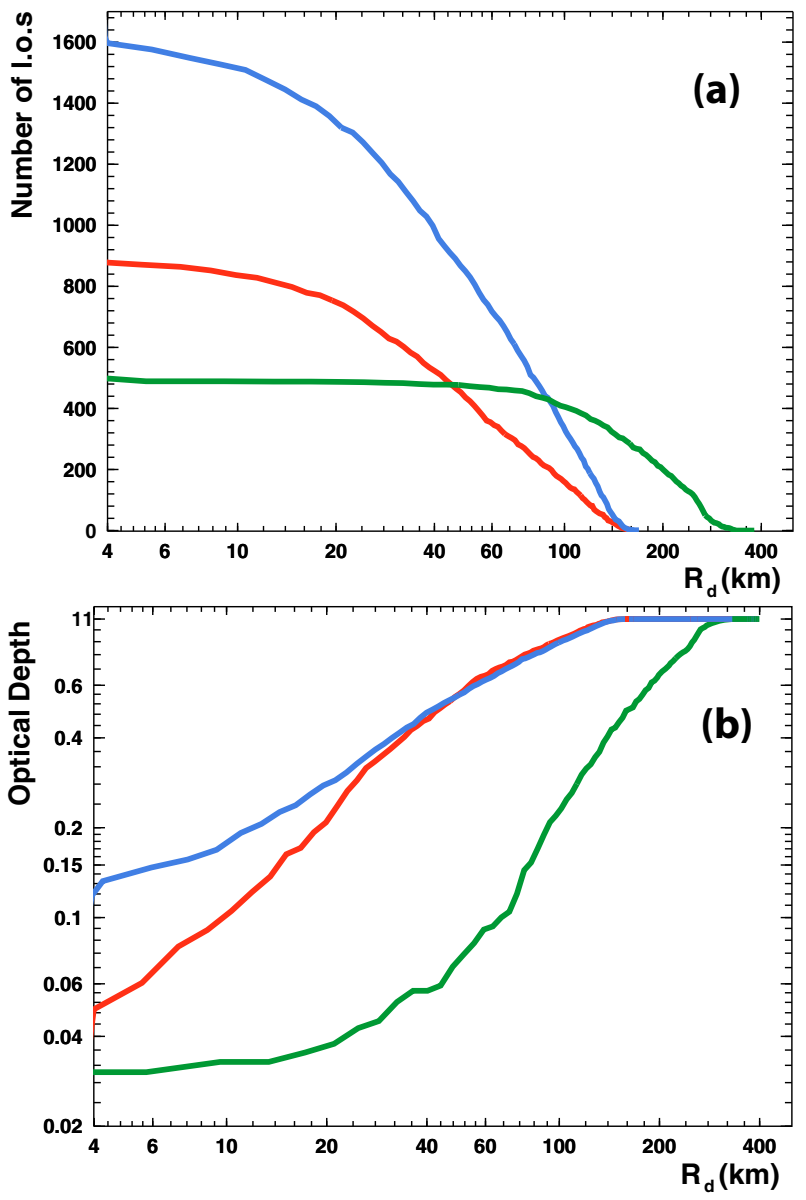

Fig. 13. a) $N_{*}\left(R_{\mathrm{d}}\right)$, the number of directions with no turbulent structure of $R_{\text {diff }}(2.16 \mu \mathrm{m})<R_{\mathrm{d}}$ along the line of sight toward the obscured regions of B68 (green), cb131 (blue) and toward the Circinus nebula (red). b) The 95\% CL maximum optical depth of structures with $R_{\text {diff }}(2.16 \mu \mathrm{m})<R_{\mathrm{d}}$.

best limits are naturally obtained toward B68, as a consequence of the better knowledge of the stellar sizes. The shape of the cumulative $R_{\mathrm{diff}}^{\mathrm{min}}$ distributions differs from the one obtained toward the SMC, because the contribution of (big) red giant stars is smaller.

We can now interpret our limits on $R_{\text {diff }}$ to constrain the global structure of the nebulae, and specifically put limits on the existence and the structure of local turbulent dense cores (cells) within the nebulae (see Lada et al. 2007; and Racca 2009).

- Probing the global structure?

If the nebula is a simple object described by a single Kolmogorov turbulence law, characterized by an outer scale of $L_{z} \sim 17000 \mathrm{AU}$ (maximum depth of gas crossed by the light in $\mathrm{B} 68$ ), then the minimum expected value of $R_{\text {diff }}$ is deduced from Eq. (1), where $\sigma_{3 n}$ is limited by $n_{\max }$ the maximum molecular density. This maximum is estimated to be $2.61 \times 10^{5} \mathrm{~cm}^{-3}$ for B68 (Hotzel et al. 2002). Using this constraint and the maximum outer scale value in (1) gives for B68

$$
\begin{aligned}
& R_{\text {diff }}(2.16 \mu \mathrm{m})> \\
& \begin{aligned}
263 \mathrm{~km}\left[\frac{2.16 \mu \mathrm{m}}{1 \mu \mathrm{m}}\right]^{\frac{6}{5}}\left[\frac{17000 \mathrm{AU}}{10 \mathrm{AU}}\right]^{-\frac{1}{5}}\left[\frac{2.61 \times 10^{5} \mathrm{~cm}^{-3}}{10^{9} \mathrm{~cm}^{-3}}\right]^{-\frac{6}{5}} \\
>3 . \times 10^{6} \mathrm{~km} .
\end{aligned}
\end{aligned}
$$

For cb131, the same calculation based on data from (Bacmann et al. 2000) gives $R_{\text {diff }}(2.16 \mu \mathrm{m})>4.2 \times 10^{6} \mathrm{~km}$. These large diffusion radii, which are owing to the weakness of the power spectrum at small scales, are much too large to induce any observable scintillation effect on any type of stars. Indeed, because $\theta_{\mathrm{s}}>\theta\left(R_{\odot}\right.$ at $\left.10 \mathrm{kpc}\right)$, Eq. (3) gives $R_{\mathrm{s}} / R_{\text {ref }}>3100$ at $\lambda=2.16 \mu \mathrm{m}$, and the expected modulation index is completely negligible (out of scale in Fig. 3).

- Probing local sub-structures?

In contrast, local turbulent dense cores with much smaller diffusion radii could potentially produce observable scintillation. Therefore our upper limits on scintillation can be interpreted as upper limits on the existence of turbulent cells with $R_{\text {diff }}(2.16 \mu \mathrm{m})<350 \mathrm{~km}$ within the volume of the nebula. Using Eq. (1), it can be interpreted as limits on the distribution of the product

$$
\left[\frac{\sigma_{3 n}}{10^{9} \mathrm{~cm}^{-3}}\right]\left[\frac{L_{z}}{10 \mathrm{AU}}\right]^{\frac{1}{6}}=\left[\frac{R_{\mathrm{diff}}(2.16 \mu \mathrm{m})}{663 \mathrm{~km}}\right]^{-\frac{5}{6}}
$$

along the lines of sight; thanks to the small exponent of $L_{z}$, a rough hypothesis for the core size would allow one to extract upper limits on the frequency of cores with a density dispersion higher than a given value $\sigma_{3 n}$.

No conclusion can be infered for structures with $R_{\text {diff }}>$ $350 \mathrm{~km}$, because they cannot produce a detectable scintillation in our sample because of our limited photometric precision on the small stars.

\section{Conclusions and perspectives}

The aim of the test was to study the feasibility of a systematic search for scintillation. We were lucky enough to find a stochastic variable light-curve that is compatible with a scintillation effect; but considering the low probability of such an event, which is related to the low density of the nebulae, a program of synchroneous multicolor and multi-epoch observations is necessary to obtain a convincing signature of the effect.

From our search for invisible gas toward the SMC, we conclude that an ambitious program using a wide field, fast readout camera at the focal plane of a $>4 \mathrm{~m}$ telescope should either discover turbulent gas in the halo, or exclude this type of hidden baryonic matter. With such a setup, significant results should be obtained with an exposure of $\sim 10^{6}$ star $\times$ hour in $V$ passband.

The hardware and software techniques required for scintillation searches are available just now, and a reasonably priced dedicated project could be operational within a few years. Alternatives under study are the use of the data from the LSST project and from the GAIA mission. If a scintillation indication is found in the future, one will have to consider a much more ambitious project involving synchronized telescopes, a few thousand kilometers apart. Such a project would allow one to temporally and spatially sample an interference pattern, unambiguously providing the refractive length scale $R_{\text {ref }}$, the speed, and the dynamics of the scattering medium.

Acknowledgements. We are grateful to the IPM for supporting F. Habibi during his stay in Tehran. We thank Prof. P. Schwemling for his help in the determination of the variable star parameters, J.-F. Glicenstein, F. Cavalier and P. Hello for their participation to discussions. We thank Prof. J.-F. Alves for providing us with the B68 absorption map. We are grateful to the referee for his constructive remarks that allowed us to significantly improve this article. 


\section{Appendix A: Connection between the diffusion radius and the gas structuration}

We consider a gaseous medium characterized by a cell with size $\left(L_{x}, L_{y}, L_{z}\right)$. An electromagnetic plane wave propagating along $z$ and crossing the medium is distorted. The distorsion is caused by the variation of the column density along the propagation of the light and can be described by a two-dimensional phase delay $\phi(x, y)$. We use the phase structure function to characterize the variations of the phase as follows:

$$
\begin{aligned}
D_{\phi}(x, y) & =\left\langle\left(\phi\left(x^{\prime}+x, y^{\prime}+y\right)-\phi\left(x^{\prime}, y^{\prime}\right)\right)^{2}\right\rangle \\
& =2\left[\left\langle\phi^{2}\left(x^{\prime}, y^{\prime}\right)\right\rangle-\left\langle\phi\left(x^{\prime}+x, y^{\prime}+y\right) \phi\left(x^{\prime}, y^{\prime}\right)\right\rangle\right] \\
& =2\left[\xi_{\phi}(0,0)-\xi_{\phi}(x, y)\right]
\end{aligned}
$$

where $\xi_{\phi}(x, y)$ is the correlation function of the screen phase and is related to the phase spectral density $S_{\phi}\left(q_{x}, q_{y}\right)$ (power spectrum per volume unit) through the Fourier transform as

$\xi_{\phi}(x, y)=\iint S_{\phi}\left(q_{x}, q_{y}\right) \mathrm{e}^{2 \pi \mathrm{i}\left(x q_{x}+y q_{y}\right)} \mathrm{d} q_{x} \mathrm{~d} q_{y}$.

Substituting Eq. (A.2) in (A.1) we obtain

$D_{\phi}(x, y)=2 \iint S_{\phi}\left(q_{x}, q_{y}\right)\left(1-\mathrm{e}^{2 \pi \mathrm{i}\left(x q_{x}+y q_{y}\right)}\right) \mathrm{d} q_{x} \mathrm{~d} q_{y}$.

The relation between the two-dimensional spectrum of the phase, $S_{\phi}\left(q_{x}, q_{y}\right)$ and the three-dimensional spectrum of the number density fluctuations has been derived for a plasma by Lovelace (Lovelace 1970; Lovelace et al. 1970). Here we extend the concept to the optical wavelength for a medium of molecular gas:

$S_{\phi}\left(q_{x}, q_{y}\right)=2 \pi L_{z}\left(\frac{(2 \pi)^{2} \alpha}{\lambda}\right)^{2} S_{3 \delta n}\left(q_{x}, q_{y}, q_{z}=0\right)$,

where $S_{3 \delta n}\left(q_{x}, q_{y}, q_{z}\right)$ is the spectrum of the density fluctuations of the molecular gas in three dimensions $(\delta n=n-\langle n\rangle), \alpha$ is the average polarizability of the molecules and $L_{z}$ is the thickness of the medium along the line of sight.

Assuming the gaseous medium to be isotropically turbulent, the $3 \mathrm{D}$ spectral density obeys a power law relation within the turbulence inertial range:

$S_{3 \delta n}\left(q_{x}, q_{y}, q_{z}\right)=C_{n}^{2} q^{-\beta} \quad L_{\text {out }}^{-1}<q<L_{\text {in }}^{-1}$,

where $q=\sqrt{q_{x}^{2}+q_{y}^{2}+q_{z}^{2}}, \beta=11 / 3$ is taken for the Kolmogorov turbulence, $C_{n}^{2}$ is the turbulence strength parameter and $L_{\text {out }}$ and $L_{\text {in }}$ are the outer and inner scales of the turbulence respectively. By substituting Eqs. (A.5) and (A.4) in Eq. (A.3), we compute the phase structure function in the polar coordinate system:

$D_{\phi}(r)=2 C_{n}^{2}(2 \pi) L_{z}\left(\frac{(2 \pi)^{2} \alpha}{\lambda}\right)^{2} \int_{0}^{\infty} \int_{0}^{2 \pi} q^{-\beta}\left(1-\mathrm{e}^{2 \pi \mathrm{i} r q \cos \theta}\right) \mathrm{d} \theta q \mathrm{~d} q$.

The integration results in

$D_{\phi}(r)=2 C_{n}^{2}(2 \pi)^{\beta} f(\beta) L_{z}\left(\frac{(2 \pi)^{2} \alpha}{\lambda}\right)^{2} r^{\beta-2}$,

where

$f(\beta)=\int_{0}^{\infty} s^{1-\beta}\left(1-J_{0}(s)\right) \mathrm{d} s=\frac{2^{-\beta} \beta \Gamma(-\beta / 2)}{\Gamma(\beta / 2)}$.

A108, page 12 of 13
For the Kolmogorov turbulence $f(\beta=11 / 3) \sim 1.118$.

We define the diffusion radius $R_{\text {diff }}$ as the transverse scale for which $D_{\phi}\left(R_{\text {diff }}\right)=1$ rad. $R_{\text {diff }}$ is directly obtained from Eq. (A.6):

$R_{\text {diff }}=\left[2 C_{n}^{2}(2 \pi)^{\beta+4} f(\beta) L_{z} \alpha^{2} \lambda^{-2}\right]^{1 /(2-\beta)}$.

We will now link the turbulence parameter $C_{n}^{2}$ to the dispersion of the density fluctuation. From Parseval's theorem the dispersion of the density fluctuation is equal to the auto-correlation function at origin:

$\sigma_{3 n}^{2}=\frac{1}{L_{x} L_{y} L_{z}} \int \delta_{n}^{2} \mathrm{~d} x \mathrm{~d} y \mathrm{~d} z=\xi(0)$.

On the other hand the auto-correlation is equal to the integration over the spectrum in the Fourier space

$\xi(0)=\int S_{3 \delta n}(q) \mathrm{d}^{3} q$

Using Eq. (A.5), the dispersion of the density fluctuations is related to $C_{n}^{2}$ as

$\sigma_{3 n}^{2}=\int_{L_{\text {out }}^{-1}}^{L_{\text {in }}^{-1}} C_{n}^{2} q^{-\beta}(4 \pi) q^{2} \mathrm{~d} q=\frac{4 \pi C_{n}^{2}}{\beta-3}\left(L_{\text {out }}^{\beta-3}-L_{\text {in }}^{\beta-3}\right)$.

Assuming $L_{z}=L_{\text {out }} \gg L_{\text {in }}$, for $\beta=11 / 3$, we estimate $C_{n}^{2}$ as

$C_{n}^{2}=\frac{\sigma_{3 n}^{2}}{6 \pi L_{z}^{2 / 3}}$

Finally by substituting Eq. (A.11) in (A.7), $R_{\text {diff }}$ is obtained in terms of the cloud's parameters and the wavelength as

$R_{\mathrm{diff}}=231.1 \mathrm{~km}\left[\frac{\alpha}{\alpha_{\mathrm{H}_{2}}}\right]^{-\frac{6}{5}}\left[\frac{\lambda}{1 \mu \mathrm{m}}\right]^{\frac{6}{5}}\left[\frac{L_{z}}{10 \mathrm{AU}}\right]^{-\frac{1}{5}}\left[\frac{\sigma_{3 n}}{10^{9} \mathrm{~cm}^{-3}}\right]^{-\frac{6}{5}}$

where $\alpha_{\mathrm{H}_{2}}=0.802 \times 10^{-24} \mathrm{~cm}^{3}$ is the polarisability of molecule $\mathrm{H}_{2}$.

\section{Appendix B: Limitations of the photometric precision}

In our photometric optimization, we found that the photometric precision on unblended stars was limited by several factors. First, the PSF of very bright stars significantly differs from the ideal Gaussian and the photometric precision on these stars is seriously affected. Second, the best photometric precision does not follow the naive expectation assuming only Poissonian noise. For stars fainter than $K_{\mathrm{s}}=14.6$ (or $J=16.6$ ) we were able to reproduce the behavior of the PSF fit $\chi^{2}$ and of the fitted flux uncertainties by assuming that the relative uncertainty $\sigma_{i, j}$ on a pixel content results from the combination of the Poissonian fluctuations of the number of photoelectrons $N_{i, j}^{\gamma \mathrm{e}}$, and of a systematic uncertainty (i.e. not changing with time, but depending on the pixel) due to the flat-fielding procedure:

$\sigma_{i, j}^{2}=\frac{1}{N_{i, j}^{\gamma \mathrm{e}}}+\left[\frac{\Delta C_{i, j}}{C_{i, j}}\right]^{2}$,

where $N_{i, j}^{\gamma \mathrm{e}}$ is the total number of photoelectrons (from the fitted star and the - usually dominant - sky background), and $\frac{\Delta C_{i, j}}{C_{i, j}}$ is the uncertainty of the flat-field coefficient for pixel $(i, j)$. From 
the comparison of two flat-fields taken at different epochs, we estimated that $\frac{\Delta C_{i, j}}{C_{i, j}} \sim 0.63 \%$ (both in $K_{\mathrm{S}}$ and in $J$ ). A third source of noise in $J$ comes from a residual fringing; we measured that fringes contribute to a $\sim 40 \%$ increase of the Poissonian fluctuation $\sqrt{N_{i, j}^{\gamma \mathrm{e}}}$ in a domain size comparable to the PSF width because of their small spatial scale.

We conclude that the contribution of the Poissonian noise dominates the error when the flux from the star plus the total sky background within the PSF fitting domain is small ( $2000 \mathrm{ADU}=10600 \gamma \mathrm{e})$. For brighter stars or for stars located in a dusty environment (producing a large sky IR background), the second - systematic - term dominates.

\section{References}

Alcock, C., Allsman, R. A., Alves, D., et al. 2000, ApJ, 542, 281

Alves, J., Lada, C. J., \& Lada, E. A. 2001, Nature, 409, 159

Ansari, R. 1996, Vistas in Astronomy, 40, No. 4

Bacmann, A., André, P., Puget, J.-L., et al. 2000, A\&A, 361, 555

Caldwell, J., \& Coulson, I. 1986, MNRAS, 218, 223

Cox, A. N. 2000, Allen's Astrophysical Quantities, 4th ed. (Springer)

De Paolis, F., Ingrosso, G., Jetzer, Ph., \& Roncadelli, M. 1995, Phys. Rev. Lett., 74,14

De Paolis, F., Ingrosso, G., Jetzer, Ph., \& Roncadelli, M. 1998, ApJ, 500, 59

Dravins, D., Lindegren, L., Mezey, E., \& Young, A. T. 1997, Pub. Ast. Soc. Pacific, 109 (parts I and II) (part III), 1998, 110

ESO-GASGANO User's Manual, Amico, P., Kornweibel, N., \& Zamparelli, M. 2001, Doc. No. VLT-PRO-ESO-19000-1932
ESO SOFI Exposure Time Calculator, Version 3.2.1. 2007, http://www. eso . org/observing/etc/

Georgelin, Y. M., Amram, P., Georgelin, Y. P., et al. 1994, A\&AS, 108, 513

Glass, I. S., Ganesh, S., Alard, C., et al. 1999, MNRAS, 308, 127

Hamadache, C., Le Guillou, L., Tisserand, P., et al. 2006, A\&A, 454, 185

Hotzel, S., Harju, J., Juvela, M., et al. 2002, A\&A, 391, 275

Johnson, H. L. 1966, ARA\&A, 4, 193

Lada, C. J., Alves, J. F., \& Lombardi, M. 2007, Near-Infrared Extinction and Molecular Cloud Structure, Protostars and Planets V, 3

Lovelace, R. V. E. 1970, Ph.D. Thesis, Cornell University

Lovelace, R. V. E., Salpeter, E. E., Sharp, L. E., \& Harris, D. E. 1970, ApJ, 159, 1047

Lyne, A. G., \& Graham-Smith., F. 1998, Pulsar Astronomy (Cambridge University Press)

Moniez, M. 2003, A\&A, 412, 105

Moniez, M. 2010, Gen. Rel. Grav., 42, 2047

Narayan, R. 1992, Phil. Trans. R. Soc. Lond. A, 341, 151

Ojha, D. K., Omont, A., Ganesh, S., et al. 2000, J. Astrophys. Astr., 21, 77

Pfenniger, D., \& Combes, F. 1994, A\&A, 285, 94

Pfenniger, D., Combes, F., \& Martinet, L. 1994, A\&A, 285, 79

Racca, G. A., Vilas-Boas, J. W. S., \& De la Reza, R. 2009, ApJ, 703, 1444

Rahal, Y. R., Afonso, C., Albert, J.-N., et al. 2009, A\&A, 500, 1027

Russeil, D., Amram, P., Georgelin, Y. P., et al. 1998, A\&AS, 130, 119

Skrutskie, M. F., Cutri, R. M., Stiening, R., et al. 2006, AJ, 131, 1163

Sterken, C., \& Jaschek, C. 1996, Light Curves of Variable Stars, a pictorial Atlas (Cambridge University Press)

Szewczyk, O., Pietrzynski, G., Gieren, W., et al. 2009, AJ, 138, 1661

Tisserand, P. 2004, Ph.D. Thesis, Université de Nice-Sophya Antipolis CEA DAPNIA-04-09-T

Tisserand, P., Le Guillou, L., Afonso, C., et al. 2007, A\&A, 469, 387

Ventsel, H. 1973, Théorie des probabilités (Moscou: Mir)

Wyrzykowski, L., Kozlowski, S., Skowron, J., et al. 2010, MNRAS, 407, 189 